\title{
Long non-coding RNA XIST: a novel oncogene in multiple cancers
}

\author{
Jun Yang ${ }^{1}$, Manlong $\mathrm{Qi}^{2}$, Xiang Fei ${ }^{3}$, Xia Wang ${ }^{3}$ and Kefeng Wang ${ }^{3 *}$ (D)
}

\begin{abstract}
Long non-coding RNA (IncRNA) X-inactive specific transcript (XIST) is an important IncRNA derived from the XIST gene in mammals. XIST is abnormally expressed in numerous tumors, in most of which XIST functions as an oncogene. XIST is involved in multiple aspects of carcinogenesis, including tumor onset, progression, and prognosis. In our review, we collected and analyzed the recent studies on the impact of XIST in human tumor development. The multilevel molecular functions of XIST in human tumors are comprehensively reviewed to clarify the pathologic mechanisms and to offer a novel direction for further study.
\end{abstract}

Keywords: Long non-coding RNA, XIST, Oncogene, Cancer

\section{Introduction}

Malignant tumors are the second leading cause of death worldwide and a threat to human health. Many studies have shown that approximately $70 \%$ of cardiovascular diseases can be cured by lifestyle adjustments. Therefore, tumors are likely to overtake cardiovascular disease as the leading cause of death within the next few years. Early detection, diagnosis, and treatment are important factors affecting the prognosis of tumors. Hence, researchers are attempting to identify the relevant novel biomarkers and prognostic factors.

Long non-coding RNAs (lncRNAs) are a cluster of RNA molecules between 200 and 100,000 nucleotides in length, and are involved in the regulation of many intracellular processes, with the exception of coding proteins (Spizzo et al. 2012). Recent studies have shown that lncRNAs are involved in X staining silencing, genomic imprinting, chromatin modification, transcriptional activation, transcriptional interference, nuclear transport, tumor regulation, and other important regulatory processes (Yue et al. 2014; Rafiee et al. 2018; Wang et al.

\footnotetext{
*Correspondence: wang.kefeng@hotmail.com

${ }^{3}$ Department of Urology, Shengjing Hospital of China Medical University, \#36 Sanhao Street, Heping, Liaoning 110004 Shenyang, China

Full list of author information is available at the end of the article
}

2017); however, the mechanism underlying lncRNA regulation of the onset and development of tumors has not been established.

LncRNA X-inactive specific transcript (XIST) was first reported by Brown et al. (1991) in 1991. XIST is a product of the XIST gene and a key regulatory factor of $\mathrm{X}$-chromosome inactivation $(\mathrm{XCI})$ in mammals. $\mathrm{XCI}$ is an epigenetic silencing of a random $\mathrm{X}$ chromosome in female cells to balance the level of X gene expression between males and females (Lyon 1972; Loda and Heard 2019). More recently, abnormal overexpression of XIST was identified in a variety of human malignant tumors, such as esophageal cancer (EC) (Wu et al. 2017a), gastric cancer (GC) (Chen et al. 2016), colorectal cancer (CRC) (Zhang et al. 2019a), pancreatic cancer (Sun et al. 2018a), hepatocellular carcinoma (HCC) (Liu and Xu 2019), laryngeal cancer (Xiao et al. 2019), lung cancer (Tantai et al. 2015), glioma (Yao et al. 2015), neuroblastoma (NB) (Zhang et al. 2019b), osteosarcoma (OS) (Li et al. 2017), bladder cancer (BC) (Xu et al. 2018a), retinoblastoma (RB) (Lyu et al. 2019), cervical cancer (CC) (Zhu et al. 2018a), thyroid cancer (Xu et al. 2018b), nasopharyngeal carcinoma (NPC) (Song et al. 2016), melanoma (Hao et al. 2021), and leukemia (Wang et al. 2020a). In our review, we have summarized the mechanism underlying original author(s) and the source, provide a link to the Creative Commons licence, and indicate if changes were made. The images or other third party material in this article are included in the article's Creative Commons licence, unless indicated otherwise in a credit line to the material. If material is not included in the article's Creative Commons licence and your intended use is not permitted by statutory regulation or exceeds the permitted use, you will need to obtain permission directly from the copyright holder. To view a copy of this licence, visit http://creativecommons.org/licenses/by/4.0/. 
XIST, as well as the clinical significance of XIST, in the occurrence and progression of tumors.

\section{XIST in various human tumors}

XIST has been shown to be abnormally overexpressed in multiple cancers, exhibiting the properties of an oncogene in promoting tumor growth, invasion, metastasis, colony formation, and chemotherapy resistance (Fang et al. 2016; Zhang et al. 2017a; Li et al. 2019a). XIST also exhibits anti-tumor properties in a small subset of tumors, such as lymphomas (Parodi 2020). In addition, XIST displays an opposite effect in the same cancer, suggesting that XIST controls cancer development at multiple levels ( $\mathrm{Ma}$ et al. 2020; Li et al. 2020a). In recent years, a number of studies have shown that XIST is involved in the clinicopathologic development of multiple cancers through post-transcriptional gene regulation. The specific mechanisms and functional characteristics of XIST in various human tumors are listed in Tables 1, 2, 3, 4, 5, 6, 7, 8 .

Table 1 Functional characterization of XIST in digestive system tumors

\begin{tabular}{|c|c|c|c|c|c|c|}
\hline Tumor types & Expression & Role & Function role & miRNAs & Related genes & References \\
\hline Esophageal cancer & Upregulation & Oncogene & Proliferation, migration, and invasion & miR-101 & $\mathrm{EZH} 2$ & Wu et al. 2017a) \\
\hline Esophageal cancer & Upregulation & Oncogene & $\begin{array}{l}\text { Proliferation, apoptosis, migration, } \\
\text { and invasion }\end{array}$ & miR-494 & CDK6/JAK2/STAT3 & Chen et al. 2019a) \\
\hline Esophageal cancer & Upregulation & Oncogene & $\begin{array}{l}\text { Cell cycle, proliferation, migration, } \\
\text { invasion, and apoptosis }\end{array}$ & miR-129-5p & CCND1 & Wang et al. 2021) \\
\hline Gastric cancer & Upregulation & Oncogene & Proliferation, migration, and invasion & miR-101 & $\mathrm{EZH} 2$ & Chen et al. 2016) \\
\hline Gastric cancer & Upregulation & Oncogene & Proliferation and invasion & miR-497 & MACC1 & Ma et al. 2017) \\
\hline Gastric cancer & Upregulation & Oncogene & Proliferation, migration, and invasion & miR-185 & TGF- $\beta 1$ & Zhang et al. 2018) \\
\hline Gastric cancer & Upregulation & Oncogene & Proliferation, migration, and invasion & miR-337 & JAK2 & Zheng et al. 2020a) \\
\hline Gastric cancer & Upregulation & Oncogene & $\begin{array}{l}\text { Proliferation, migration, and apop- } \\
\text { tosis }\end{array}$ & miR-132 & PXN & Li et al. 2020b) \\
\hline Colorectal cancer & Upregulation & Oncogene & Proliferation & miR-132-3p & MAPK1 & Song et al. 2017) \\
\hline Colorectal cancer & Upregulation & Oncogene & $\begin{array}{l}\text { Proliferation, migration, invasion, } \\
\text { emt, and stem cell formation }\end{array}$ & miR-200b-3p & ZEB1 & Chen et al. 2017) \\
\hline Colorectal cancer & Upregulation & Oncogene & Migration and invasion & miR-137 & $\mathrm{EZH} 2$ & Liu et al. 2018) \\
\hline Colorectal cancer & Upregulation & Oncogene & Proliferation and invasion & miR-34a & WNT1 & Sun et al. 2018b) \\
\hline Colorectal cancer & Upregulation & Oncogene & Growth, viability, apoptosis, and emt & $\operatorname{miR}-486 b-5 p$ & $\mathrm{NRP}-2$ & Liu et al. 2019) \\
\hline Colorectal cancer & Upregulation & Oncogene & $\begin{array}{l}\text { Migration, proliferation, emt, and } \\
\text { apoptosis }\end{array}$ & miR-93-5p & HIF-1A & Yang et al. 2020) \\
\hline Colorectal cancer & Upregulation & Oncogene & $\begin{array}{l}\text { Proliferation, migration, invasion, } \\
\text { and apoptosis }\end{array}$ & miR-338-3p & PAX5 & Li et al. 2021) \\
\hline Colorectal cancer & Upregulation & Oncogene & $\begin{array}{l}\text { Proliferation, migration, invasion, } \\
\text { and apoptosis }\end{array}$ & miR-497-5p & FOXK1 & Wang et al. 2020b) \\
\hline Colorectal cancer & Upregulation & Oncogene & Chemoresistance & miR-124 & SGK1 & Zhu et al. 2018b) \\
\hline Colorectal cancer & Upregulation & Oncogene & $\begin{array}{l}\text { Viability, proliferation, apoptosis, and } \\
\text { chemoresistance }\end{array}$ & $\operatorname{miR}-30 a-5 p$ & ROR1 & Zhang et al. 2019c) \\
\hline Colorectal cancer & Upregulation & Oncogene & $\begin{array}{l}\text { Proliferation, migration, invasion, } \\
\text { and chemoresistance }\end{array}$ & miR-137 & PKM2/PKM1 & Zheng et al. 2020) \\
\hline Pancreatic cancer & Upregulation & Oncogene & Proliferation, migration, and invasion & $\operatorname{miR}-34 a-5 p$ & / & Sun et al. 2018a) \\
\hline Pancreatic cancer & Upregulation & Oncogene & Proliferation & miR-133a & EGFR & Wei et al. 2017) \\
\hline Pancreatic cancer & Upregulation & Oncogene & Proliferation & miR-140/124 & iASPP & Liang et al. 2017) \\
\hline Pancreatic cancer & Upregulation & Oncogene & Emt & miR-34a & YAP & Zou et al. 2020) \\
\hline Pancreatic cancer & Upregulation & Oncogene & Migration, invasion, and emt & miR-429 & ZEB1 & Shen et al. 2019) \\
\hline Pancreatic cancer & Upregulation & Oncogene & Proliferation, migration, and invasion & miR-141-3p & TGF- $\beta 2$ & Sun and Zhang 2019) \\
\hline Pancreatic cancer & Upregulation & Oncogene & Proliferation & miR-137 & Notch 1 & Liu et al. 2020) \\
\hline Hepatocellular carcinoma & Upregulation & Oncogene & Proliferation & miR-200b-3p & / & Liu and Xu 2019) \\
\hline Hepatocellular carcinoma & Upregulation & Oncogene & Proliferation and apoptosis & $\operatorname{miR}-488$ & / & Dong et al. 2020) \\
\hline Hepatocellular carcinoma & Upregulation & Oncogene & Proliferation and apoptosis & miR-139-5p & PDK1 & Mo et al. 2017) \\
\hline Hepatocellular carcinoma & Upregulation & Oncogene & Proliferation, migration, and invasion & miR-194-5p & MAPK1 & Kong et al. 2018) \\
\hline
\end{tabular}


Table 2 Main characteristics of the studies included in the review of digestive system tumors

\begin{tabular}{|c|c|c|c|c|c|c|c|c|c|}
\hline Study & Tumor types & $\begin{array}{l}\text { Sample size } \\
\text { (Normal:Tumor) }\end{array}$ & $\begin{array}{l}\text { Detection } \\
\text { Method }\end{array}$ & $P$ value & $\begin{array}{l}\text { TNM } \\
\text { ( } p \text { value) }\end{array}$ & $\begin{array}{l}\text { LNM } \\
\text { ( } p \text { value) }\end{array}$ & $\begin{array}{l}\text { DM } \\
\text { ( } p \text { value) }\end{array}$ & $\begin{array}{l}\text { OS } \\
\text { ( } p \text { value) }\end{array}$ & References \\
\hline Wu & Esophageal cancer & $(127: 127)$ & qRT-PCR & $p=0.0092$ & $p=0.000$ & / & / & $p=0.005$ & Wu et al. 2017a) \\
\hline Chen & Esophageal cancer & (78: 78) & qRT-PCR & $p<0.001$ & / & l & / & / & Chen et al. 2019a) \\
\hline Wang & Esophageal cancer & $(42: 42)$ & qRT-PCR & $p<0.001$ & $p=0.0064$ & / & / & $p=0.0039$ & Wang et al. 2021) \\
\hline Chen & Gastric cancer & (106: 106) & qRT-PCR & $p<0.001$ & / & / & $p=0.033$ & $p=0.002$ & Chen et al. 2016) \\
\hline $\mathrm{Ma}$ & Gastric cancer & (98: 98) & qRT-PCR & $p<0.05$ & $p=0.005$ & $p=0.002$ & / & $p<0.05$ & Ma et al. 2017) \\
\hline $\mathrm{Li}$ & Gastric cancer & $(65: 65)$ & qRT-PCR & $p<0.05$ & / & / & / & / & Li et al. 2020b) \\
\hline $\mathrm{Li}$ & Gastric cancer & (98: 98) & qRT-PCR & $p<0.05$ & $p=0.0077$ & $p=0.014$ & / & / & Li et al. 2020) \\
\hline Zhang & Colorectal cancer & (196: 196) & qRT-PCR & $p<0.001$ & / & / & $p<0.001$ & $p<0.001$ & Zhang et al. 2019a) \\
\hline Yu & Colorectal cancer & $(41: 94)$ & qRT-PCR & $p<0.05$ & / & / & / & $p<0.001$ & Yu et al. 2020) \\
\hline Yang & Colorectal cancer & $(37: 37)$ & qRT-PCR & $p<0.0001$ & / & / & $p=0.008$ & / & Yang et al. 2020) \\
\hline Song & Colorectal cancer & $(50: 50)$ & qRT-PCR & $p<0.05$ & $p=0.034$ & / & / & / & Song et al. 2017) \\
\hline Chen & Colorectal cancer & $(115: 115)$ & qRT-PCR & $p<0.05$ & / & / & / & $p=0.01$ & Chen et al. 2017) \\
\hline Liu & Colorectal cancer & $(20: 20)$ & qRT-PCR & $p<0.001$ & / & / & / & / & Liu et al. 2018) \\
\hline Sun & Colorectal cancer & (120: 120) & qRT-PCR & $p<0.01$ & $p=0.005$ & $p=0.035$ & $p=0.02$ & $p<0.05$ & Sun et al. 2018b) \\
\hline Liu & Colorectal cancer & $(317: 317)$ & qRT-PCR & $p<0.05$ & $p=0.04$ & $p<0.001$ & / & $p<0.001$ & Liu et al. 2019) \\
\hline Yang & Colorectal cancer & $(36: 36)$ & qRT-PCR & $p<0.001$ & $p=0.0333$ & / & / & / & Yang et al. 2020) \\
\hline $\mathrm{Li}$ & Colorectal cancer & (30: 30$)$ & qRT-PCR & $p<0.05$ & $p=0.028$ & / & / & / & Li et al. 2021) \\
\hline Wang & Colorectal cancer & $(54: 54)$ & qRT-PCR & $p<0.01$ & / & / & / & / & Wang et al. 2020b) \\
\hline Zhang & Colorectal cancer & $(294: 294)$ & qRT-PCR & $p<0.05$ & / & $p=0.037$ & / & $p<0.001$ & Zhang et al. 2019c) \\
\hline Sun & Pancreatic cancer & (139: 139) & qRT-PCR & $p<0.001$ & / & / & / & $p<0.001$ & Sun et al. 2018a) \\
\hline Wei & Pancreatic cancer & $(64: 64)$ & qRT-PCR & $p<0.01$ & $p=0.023$ & / & / & $p=0.002$ & Wei et al. 2017) \\
\hline Liang & Pancreatic cancer & $(73: 73)$ & qRT-PCR & $p<0.01$ & / & / & / & $p=0.003$ & Liang et al. 2017) \\
\hline Shen & Pancreatic cancer & (120: 120) & qRT-PCR & $p<0.001$ & / & / & / & / & Shen et al. 2019) \\
\hline Sun & Pancreatic cancer & (30: 30$)$ & qRT-PCR & $p<0.01$ & / & / & / & / & $\begin{array}{l}\text { Sun and Zhang } \\
\text { 2019) }\end{array}$ \\
\hline Liu & Pancreatic cancer & $(40: 40)$ & qRT-PCR & $p<0.001$ & / & I & / & / & Liu et al. 2020) \\
\hline Liu & $\begin{array}{l}\text { Hepatocellular } \\
\text { carcinoma }\end{array}$ & $(55: 55)$ & qRT-PCR & $p<0.05$ & / & / & / & / & Liu and Xu 2019) \\
\hline Dong & $\begin{array}{l}\text { Hepatocellular } \\
\text { carcinoma }\end{array}$ & $(69: 69)$ & qRT-PCR & $p<0.001$ & / & / & / & / & Dong et al. 2020) \\
\hline Mo & $\begin{array}{l}\text { Hepatocellular } \\
\text { carcinoma }\end{array}$ & (88: 88) & qRT-PCR & $p<0.05$ & / & / & / & / & Mo et al. 2017) \\
\hline Kong & $\begin{array}{l}\text { Hepatocellular } \\
\text { carcinoma }\end{array}$ & $(52: 52)$ & qRT-PCR & $p<0.05$ & / & / & / & $p<0.05$ & Kong et al. 2018) \\
\hline
\end{tabular}

\section{The role of XIST in digestive system tumors $X I S T$ in esophageal cancer (EC)}

EC ranked 7th in morbidity (604,100 new cases) and mortality (544,076 deaths) worldwide in 2020 , with $70 \%$ of cases occurring in men (Sung et al. 2021). Esophageal squamous cell carcinoma (ESCC) is the histological type most common in low income Asian and African countries. The main causes of ESCC may be nutritional deficiencies, nitrosamine use, betel nut chewing, and consumption of preserved vegetables (Sung et al. 2021). Esophageal adenocarcinoma is most common in Western high income countries and is mainly caused by alcohol abuse, cigarette smoking, overweight, gastroesophageal reflux disease, and Barrett's esophagus (Sung et al. 2021). Therefore, further study of the molecular mechanism underlying EC is warranted.

Wu et al. (2017a) reported that XIST is upregulated in ESCCs and performed an oncogenic progression through regulation of the miR-101/EZH2 signal pathway (Fig. 1A). Then, another group reported that XIST induced carcinogenesis through the miR-494/CDK6/ JAK2/STAT3 signal pathway in EC, which provided a potential means for investigation of EC (Fig. 1B) (Chen et al. 2019a). Additionally, Wang et al. (2021) showed that downregulation of XIST suppressed the malignant behaviors in part by antagonizing the miR-129-5p/ CCND1 signal pathway in EC (Fig. 1C). 
Table 3 Functional characterization of XIST in respiratory system tumors

\begin{tabular}{|c|c|c|c|c|c|c|}
\hline Tumor types & Expression & Role & Function role & miRNAs & Related genes & References \\
\hline Laryngeal cancer & Upregulation & Oncogene & Proliferation, migration, and invasion & miR-124 & $\mathrm{EZH} 2$ & Xiao et al. 2019) \\
\hline Laryngeal cancer & Upregulation & Oncogene & Proliferation, migration, invasion, and apoptosis & miR-144 & IRS1 & Cui et al. 2020) \\
\hline Laryngeal cancer & Upregulation & Oncogene & Proliferation, migration, invasion, and apoptosis & miR-125b-5p & TRIB2 & Liu et al. 2020) \\
\hline Lung cancer & Upregulation & Oncogene & Proliferation, invasion, and apoptosis & miR-186-5p & / & Wang et al. 2017b) \\
\hline Lung cancer & Upregulation & Oncogene & Proliferation, migration, invasion, and apoptosis & miR-449a & $\mathrm{BCL} 2$ & Zhang et al. 2017b) \\
\hline Lung cancer & Upregulation & Oncogene & Proliferation and apoptosis & miR-140 & iASPP & Tang et al. 2017) \\
\hline Lung cancer & Upregulation & Oncogene & Growth and motility & miR-374a & LARP1 & Xu et al. 2017) \\
\hline Lung cancer & Upregulation & Oncogene & Proliferation and emt & miR-137 & Notch1 & Wang et al. 2018) \\
\hline Lung cancer & Upregulation & Oncogene & Proliferation and invasion & miR-744 & RING1 & Wang et al. 2019) \\
\hline Lung cancer & Upregulation & Oncogene & Proliferation and apoptosis & miR-335 & SOD2 & Liu et al. 2019) \\
\hline Lung cancer & Upregulation & Oncogene & Proliferation, migration, and cell cycle & miR-16 & CDK8 & Zhou et al. 2019) \\
\hline Lung cancer & Upregulation & Oncogene & Proliferation, migration, invasion, and emt & $\operatorname{miR}-212-3 p$ & CBLL1 & Qiu et al. 2019) \\
\hline Lung cancer & Upregulation & Oncogene & Proliferation, migration, invasion, and apoptosis & miR-363-3p & MDM2 & Rong et al. 2020) \\
\hline Lung cancer & Upregulation & Oncogene & Proliferation, migration, invasion, and apoptosis & miR-142-5p & PAX6 & Jiang et al. 2020) \\
\hline Lung cancer & Upregulation & Oncogene & Chemoresistance & let-7i & BAG-1 & Sun et al. 2017) \\
\hline Lung cancer & Upregulation & Oncogene & Autophagy and chemoresistance & miR-17 & ATG7 & Sun et al. 2017) \\
\hline Lung cancer & Upregulation & Oncogene & $\begin{array}{l}\text { Proliferation, migration, apoptosis, and chemore- } \\
\text { sistance }\end{array}$ & miR-144-3p & MDR1 & Tian et al. 2019) \\
\hline
\end{tabular}

Table 4 Main characteristics of the studies included in the review of respiratory system tumors

\begin{tabular}{|c|c|c|c|c|c|c|c|c|c|}
\hline Study & Tumor types & $\begin{array}{l}\text { Sample size } \\
\text { (Normal: Tumor) }\end{array}$ & Detection Method & $P$ value & $\begin{array}{l}\text { TNM } \\
\text { ( } p \text { value) }\end{array}$ & $\begin{array}{l}\text { LNM } \\
\text { ( } p \text { value) }\end{array}$ & $\begin{array}{l}\text { DM } \\
\text { ( } p \text { value) }\end{array}$ & $\begin{array}{l}\text { OS } \\
\text { ( } p \text { value) }\end{array}$ & References \\
\hline Cui & Laryngeal cancer & $(48: 48)$ & qRT-PCR & $p<0.01$ & / & / & / & / & Cui et al. 2020) \\
\hline Liu & Laryngeal cancer & $(40: 40)$ & qRT-PCR & $p<0.05$ & $p=0.005$ & / & $p=0.011$ & $p=0.0423$ & Liu et al. 2020) \\
\hline Wang & Lung cancer & (30: 30$)$ & qRT-PCR & $p<0.001$ & / & / & / & / & Wang et al. 2017b) \\
\hline Wang & Lung cancer & (33: 33$)$ & qRT-PCR & $p<0.05$ & / & / & / & / & Wang et al. 2018) \\
\hline Wang & Lung cancer & $(20: 20)$ & qRT-PCR & $p<0.001$ & $p=0.000$ & / & / & $p=0.0264$ & Wang et al. 2019) \\
\hline Liu & Lung cancer & $(45: 45)$ & qRT-PCR & $p<0.01$ & / & / & / & / & Liu et al. 2019) \\
\hline Zhou & Lung cancer & $(15: 15)$ & qRT-PCR & $p<0.05$ & / & / & / & / & Zhou et al. 2019) \\
\hline Qiu & Lung cancer & $(33: 33)$ & qRT-PCR & $p<0.05$ & / & / & / & $p<0.05$ & Qiu et al. 2019) \\
\hline Rong & Lung cancer & (35: 35$)$ & qRT-PCR & $p<0.05$ & / & / & / & / & Rong et al. 2020) \\
\hline Jiang & Lung cancer & $(30: 30)$ & qRT-PCR & $p<0.05$ & / & / & / & / & Jiang et al. 2020) \\
\hline Sun & Lung cancer & $(50: 50)$ & qRT-PCR & $p<0.05$ & $p=0.045$ & / & / & / & Sun et al. 2017) \\
\hline $\mathrm{Xu}$ & Lung cancer & $(30: 30)$ & qRT-PCR & $p<0.05$ & / & / & / & / & Xu et al. 2020) \\
\hline
\end{tabular}

Thus, XIST provides a molecular mechanism for inhibiting EC progression.

\section{XIST in gastric cancer (GC)}

It is estimated that GC caused 1,089,103 new cases and 768,793 deaths in 2020, ranking 6th in incidence and 4th in mortality globally (Sung et al. 2021). The main causes of GC are Helicobacter pylori infection, alcohol consumption, low fruit intake, tobacco smoking, and high consumption of processed meat (Sung et al. 2021). Thus, it is important to search for diagnostic and therapeutic strategies to improve GC patient outcomes.
Chen et al. ( 2016) reported that XIST was responsible for the aggressive phenotype of GC and was involved in post-transcriptional control via the miR-101/EZH2 signal pathway (Fig. 1A). Subsequently, Ma et al. (2017) reported that XIST promoted cell growth and invasion via miR-497/MACC1 signals, which suggested a potential prognostic factor for GC (Fig. 1D). Another research group demonstrated that XIST functioned as a competing endogenous RNA (ceRNA) to modulate TGF$\beta 1$ expression by sequestering miR-185 in GC (Fig. 1E) (Zhang et al. 2018). In addition, Zheng et al. (2020a) showed that XIST silencing inhibited cell growth and 
Table 5 Functional characterization of XIST in nervous system tumors

\begin{tabular}{|c|c|c|c|c|c|c|}
\hline Tumor types & Expression & Role & Function role & miRNAs & Related genes & References \\
\hline Glioma & Upregulation & Oncogene & Proliferation, migration, invasion, and apoptosis & miR-152 & / & Yao et al. 2015) \\
\hline Glioma & Upregulation & Oncogene & Tumorigenicity and angiogenesis & $\operatorname{miR}-429$ & / & Cheng et al. 2017) \\
\hline Glioma & Upregulation & Oncogene & Angiogenesis & miR-137 & $\mathrm{ZO}-2 / \mathrm{FOXC1}$ & Yu et al. 2017) \\
\hline Glioma & Upregulation & Oncogene & Proliferation & miR-137 & Rac1 & Wang et al. 2017c) \\
\hline Glioma & Upregulation & Oncogene & $\begin{array}{l}\text { Viability, migration, invasion, apoptosis, and glucose } \\
\text { metabolism }\end{array}$ & $\operatorname{miR}-126$ & IRS1/PI3K/Akt & Cheng et al. 2020) \\
\hline Glioma & Upregulation & Oncogene & Proliferation, metastasis, and emt & miR-133a & $\mathrm{SOX} 4$ & Luo et al. 2020) \\
\hline Glioma & Upregulation & Oncogene & Proliferation, migration, invasion, and apoptosis & miR-204-5p & $\mathrm{BCl}-2$ & Shen et al. 2020) \\
\hline Glioma & Upregulation & Oncogene & Stemness & miR-152 & KLF4 & Gong et al. 2021) \\
\hline Glioma & Upregulation & Oncogene & Proliferation, invasion, apoptosis, and radio-sensitivity & miR-329-3p & CREB1 & Wang et al. 2020c) \\
\hline Neuroblastoma & Upregulation & Oncogene & Cell cycle, proliferation, and radio-sensitivity & $\operatorname{miR}-375$ & L1CAM & Yang et al. 2020) \\
\hline
\end{tabular}

Table 6 Main characteristics of the studies included in the review of nervous system tumors

\begin{tabular}{|c|c|c|c|c|c|c|c|c|c|}
\hline Study & Tumor types & $\begin{array}{l}\text { Sample size } \\
\text { (Normal: Tumor) }\end{array}$ & Detection Method & $P$ value & $\begin{array}{l}\text { TNM } \\
\text { ( } p \text { value) }\end{array}$ & $\begin{array}{l}\text { LNM } \\
\text { ( } p \text { value) }\end{array}$ & $\begin{array}{l}\text { DM } \\
\text { ( } p \text { value) }\end{array}$ & $\begin{array}{l}\text { OS } \\
\text { ( } p \text { value) }\end{array}$ & References \\
\hline Wang & Glioma & $(18: 30)$ & qRT-PCR & $p<0.05$ & $p=0.014$ & / & / & / & Wang et al. 2017c) \\
\hline $\mathrm{Du}$ & Glioma & $(69: 69)$ & qRT-PCR & $p<0.01$ & $p=0.079$ & / & / & $p=0.0007$ & Du et al. 2017) \\
\hline Wang & Glioma & $(30: 30)$ & qRT-PCR & $p<0.05$ & / & / & / & / & Wang et al. 2020c) \\
\hline Zhang & Neuroblastoma & $(30: 30)$ & qRT-PCR & $p<0.05$ & $p=0.011$ & / & / & / & Zhang et al. 2019b) \\
\hline Yang & Neuroblastoma & $(20: 36)$ & qRT-PCR & $p<0.05$ & / & / & / & / & Yang et al. 2020) \\
\hline
\end{tabular}

migration via regulation of the miR-337/JAK2 signal pathway in GC (Fig. 1F). Li et al. (2020b) showed that XIST increased PXN expression through combination with miR-132 (Fig. 1G). Knockdown of XIST reversed the anti-tumor effect exerted by PXN, which highlighted the therapeutic role in GC. Six months later, Li et al. (2020) suggested that XIST promoted the progression of GC via upregulation of HNF4A enrichment in the promoter region of EPHA1.

Taken together, the above data indicated that XIST was responsible for an oncogene in GC development.

\section{XIST in colorectal cancer (CRC)}

It has been estimated that there will be $1,880,725$ new cases of CRC and 915,880 deaths in 2020, accounting for approximately one in 10 new cases and deaths (Sung et al. 2021). CRC ranks third in morbidity, but second in mortality (Sung et al. 2021). Reducing physical activity, gaining excess weight, drinking too much alcohol, smoking cigarettes, and eating red or processed meat are risk factors for CRC, while calcium supplementation and adequate intake of whole grains, fiber, and dairy products appear to reduce the risk (Siegel et al. 2020). Therefore, the pathogenesis underlying CRC is essential for identifying the therapeutic targets.
Zhang et al. (2019a) analyzed the correlation between XIST expression and the clinicopathological features of CRC. It has been reported that XIST was elevated in CRC and was used as an independent risk factor for the prognosis of CRC. Similar results indicated that patients with high XIST expression had worse survival rates, higher lymphatic metastases, shorter life cycles, and lower differentiation than patients with low XIST expression. Therefore, serum XIST expression may contribute to the diagnosis and prognosis of CRC (Yu et al. 2020). Another study concluded that METTLE14 downregulated and increased XIST expression by m6A-YTHDF2 to promote cell growth and invasion in CRC (Yang et al. 2020).

The study conducted by Song et al. (2017) indicated that XIST promoted cell growth by suppressing the miR-132-3p/MAPK1 signal pathway and was therefore a potential oncogenic target of CRC (Fig. 1H). Subsequently, the role of IncRNA-miRNA-mRNA axis in CRC became the focus of research by a number of groups. Specifically, it was shown that XIST promoted CRC cell malignant activities and upregulated ZEB1 (Fig. 1I), EZH2 (Fig. 1J), WNT1 (Fig. 1K), NRP-2 (Fig. 1L), HIF-1A (Fig. 1M), PAX5 (Fig. 1N), and FOXK1 (Fig. 1O) by modulating miR-200b-3p (Chen et al. 2017), miR-137 (Liu et al. 2018), miR-34a (Sun et al. 2018b), miR-486-5p (Liu et al. 2019), miR-93-5p (Yang et al. 2020), miR-338-3p 
Table 7 Functional characterization of XIST in other system tumors

\begin{tabular}{|c|c|c|c|c|c|c|}
\hline Tumor types & Expression & Role & Function role & miRNAs & Related genes & References \\
\hline Osteosarcoma & Upregulation & Oncogene & Proliferation and invasion & miR-137 & / & Li et al. 2019b) \\
\hline Osteosarcoma & Upregulation & Oncogene & Proliferation and invasion & miR-320b & RAP2B & Lv et al. 2018) \\
\hline Osteosarcoma & Upregulation & Oncogene & Proliferation and invasion & miR-193a-3p & RSF1 & Wu et al. 2017b) \\
\hline Osteosarcoma & Upregulation & Oncogene & Proliferation, autophagy, and apoptosis & miR-375-3p & $\mathrm{AKT} / \mathrm{mTOR}$ & Sun et al. 2019) \\
\hline Osteosarcoma & Upregulation & Oncogene & Migration, invasion, and emt & miR-153 & SNAIL & Wen et al. 2020) \\
\hline Osteosarcoma & Upregulation & Oncogene & Proliferation and apoptosis & miR-124-3p & ¡ASPP & Hai et al. 2021) \\
\hline Bladder cancer & Upregulation & Oncogene & Clone formation and emt & miR-200c & / & Xu et al. 2018a) \\
\hline Bladder cancer & Upregulation & Oncogene & Proliferation and migration & miR-133a & / & Zhou et al. 2019) \\
\hline Bladder cancer & Upregulation & Oncogene & Proliferation, migration, and invasion & miR-124 & $A R$ & Xiong et al. 2017) \\
\hline Bladder cancer & Upregulation & Oncogene & $\begin{array}{l}\text { Proliferation, migration, invasion, and } \\
\text { apoptosis }\end{array}$ & miR-139-5p & Wnt/ $\beta$-catenin & Hu et al. 2017) \\
\hline Bladder cancer & Upregulation & Oncogene & Proliferation, migration, invasion, and emt & miR-335 & / & Chen et al. 2020) \\
\hline Retinoblastoma & Upregulation & Oncogene & Proliferation, invasion, apoptosis, and emt & miR-142-5p & / & Xu and Tian 2020) \\
\hline Retinoblastoma & Upregulation & Oncogene & Proliferation, cell cycle, and apoptosis & miR-124 & STAT3 & Hu et al. 2018) \\
\hline Retinoblastoma & Upregulation & Oncogene & $\begin{array}{l}\text { Proliferation, migration, invasion, apoptosis, } \\
\text { and emt }\end{array}$ & miR-101 & ZEB1/ZEB2 & Cheng et al. 2019) \\
\hline Retinoblastoma & Upregulation & Oncogene & Proliferation and invasion & miR-140-5p & SOX4 & Wang et al. 2020d) \\
\hline Retinoblastoma & Upregulation & Oncogene & Proliferation, invasion, apoptosis, and emt & miR-200a-3p & NRP1 & Zhao et al. 2020) \\
\hline Retinoblastoma & Upregulation & Oncogene & $\begin{array}{l}\text { Proliferation, migration, invasion, autophagy, } \\
\text { and apoptosis }\end{array}$ & $\operatorname{miR}-361-3 p$ & STX17 & Yang et al. 2020) \\
\hline Retinoblastoma & Upregulation & Oncogene & Proliferation, autophagy, and drug sensitivity & miR-204-5p & / & Yao et al. 2020) \\
\hline Cervical cancer & Upregulation & Oncogene & Proliferation, apoptosis, invasion, and emt & miR-200a & Fus & Zhu et al. 2018a) \\
\hline Cervical cancer & Upregulation & Oncogene & Proliferation and apoptosis & miR-140-5p & ORC1 & Chen et al. 2019b) \\
\hline Cervical cancer & Upregulation & Oncogene & $\begin{array}{l}\text { Proliferation, migration, invasion, and } \\
\text { apoptosis }\end{array}$ & miR-889-3p & SIX1 & Liu et al. 2020) \\
\hline Thyroid cancer & Upregulation & Oncogene & Proliferation, migration, and invasion & miR-141 & / & Xu et al. 2018b) \\
\hline Thyroid cancer & Upregulation & Oncogene & Proliferation & miR-34a & MET-PI3K-AKT & Liu et al. 2018) \\
\hline Thyroid cancer & Upregulation & Oncogene & Migration and invasion & miR-101-3p & CLDN1 & Du et al. 2021) \\
\hline Nasopharyngeal cancer & Upregulation & Oncogene & Growth & $\operatorname{miR}-34 a-5 p$ & $\mathrm{E} 2 \mathrm{~F} 3$ & Song et al. 2016) \\
\hline Nasopharyngeal cancer & Upregulation & Oncogene & Proliferation and radiosensitivity & miR-29c & / & Han et al. 2017) \\
\hline Nasopharyngeal cancer & Upregulation & Oncogene & Proliferation, invasion, and apoptosis & miR-491-5p & / & Cheng et al. 2018) \\
\hline Nasopharyngeal cancer & Upregulation & Oncogene & Proliferation, metastasis, and emt & miR-148a-3p & ADAM17 & Shi et al. 2020) \\
\hline Nasopharyngeal cancer & Upregulation & Oncogene & Glycolysis, migration, and invasion & $\operatorname{miR}-381-3 p$ & NEK5 & Zhao et al. 2020) \\
\hline Melanoma & Upregulation & Oncogene & Proliferation and apoptosis & $\operatorname{miR}-23 a-3 p$ & GINS2 & Hao et al. 2021) \\
\hline Leukemia & Upregulation & Oncogene & Drug resistance, viability, and apoptosis & miR-29a & MYC & Wang et al. 2020a) \\
\hline
\end{tabular}

(Li et al. 2021), and miR-497-5p (Wang et al. 2020b), respectively.

In addition, XIST affects chemotherapy resistance of CRC. Xiao et al. (2017) showed that XIST was involved in 5-fluorouracil (5FU) resistance by promoting thymidylate synthase expression, thus XIST silencing is a potentially new therapeutic strategy to defeat 5FU resistance in patients with CRC. Corollary studies showed that XIST played a regulatory role in doxorubicin resistance via the miR-124/SGK1 signal pathway, which provided a novel way to thwart chemotherapy resistance in CRC (Fig. 1P) (Zhu et al. 2018b). Similarly,
Zhang et al. (2019c) concluded that XIST influenced chemosensitivity via the miR-30a-5p/ROR1 axis and atractylenolide II enhanced the chemotherapeutic sensitivity of CRC cells (Fig. 1Q). Moreover, Zheng et al. (2020) demonstrated that XIST/miR-137 signals enhanced glycolysis and chemotherapy tolerance of CRC by increasing the PKM2-to-PKM1 ratio, thus providing another option for improving the efficacy of chemotherapy in CRC patients (Fig. 1R).

Overall, these data supported the essential role of XIST in CRC carcinogenesis, representing novel diagnostic and therapeutic targets. 
Table 8 Main characteristics of the studies included in the review of other system tumors

\begin{tabular}{|c|c|c|c|c|c|c|c|c|c|}
\hline Study & Tumor types & $\begin{array}{l}\text { Sample size } \\
\text { (Normal:Tumor) }\end{array}$ & Detection Method & $P$ value & $\begin{array}{l}\text { TNM } \\
\text { ( } p \text { value) }\end{array}$ & $\begin{array}{l}\text { LNM } \\
\text { ( } p \text { value) }\end{array}$ & $\begin{array}{l}\text { DM } \\
\text { ( } p \text { value) }\end{array}$ & $\begin{array}{l}\text { OS } \\
\text { ( } p \text { value) }\end{array}$ & References \\
\hline $\mathrm{Li}$ & Osteosarcoma & (145: 145) & qRT-PCR & $p<0.01$ & $p=0.007$ & / & $p=0.008$ & $p<0.001$ & Li et al. 2017) \\
\hline $\mathrm{Xu}$ & Osteosarcoma & $(66: 66)$ & qRT-PCR & $p<0.01$ & / & / & / & / & Xu et al. 2017) \\
\hline Wang & Osteosarcoma & $(64: 64)$ & qRT-PCR & $p<0.01$ & / & / & $p=0.012$ & $p=0.034$ & Wang et al. 2019) \\
\hline $\mathrm{Li}$ & Osteosarcoma & $(35: 35)$ & qRT-PCR & $p<0.01$ & / & / & / & / & Li et al. 2019b) \\
\hline LV & Osteosarcoma & $(34: 34)$ & qRT-PCR & $p<0.05$ & / & / & / & / & Lv et al. 2018) \\
\hline Wen & Osteosarcoma & (30: 30$)$ & qRT-PCR & $p<0.001$ & / & / & / & / & Wen et al. 2020) \\
\hline Hai & Osteosarcoma & $(15: 38)$ & qRT-PCR & $p<0.001$ & / & / & / & $p=0.0221$ & Hai et al. 2021) \\
\hline Zhou & Bladder cancer & $(52: 52)$ & qRT-PCR & $p<0.01$ & $p=0.001$ & $p=0.001$ & $p=0.001$ & $p=0.039$ & Zhou et al. 2019) \\
\hline Xiong & Bladder cancer & $(67: 67)$ & qRT-PCR & $p<0.01$ & $p=0.01$ & / & / & / & Xiong et al. 2017) \\
\hline $\mathrm{Hu}$ & Bladder cancer & $(52: 52)$ & qRT-PCR & $p<0.05$ & $p=0.012$ & $p=0.042$ & / & $p=0.0332$ & Hu et al. 2017) \\
\hline $\mathrm{Xu}$ & Retinoblastoma & $(53: 87)$ & qRT-PCR & $p<0.05$ & / & / & / & $p<0.05$ & Xu and Tian 2020) \\
\hline $\mathrm{Hu}$ & Retinoblastoma & $(6: 30)$ & qRT-PCR & $p<0.01$ & $p<0.01$ & / & / & / & Hu et al. 2018) \\
\hline Cheng & Retinoblastoma & $(7: 53)$ & qRT-PCR & $p<0.05$ & / & / & / & / & Cheng et al. 2019) \\
\hline Wang & Retinoblastoma & $(8: 20)$ & qRT-PCR & $p<0.001$ & / & / & / & / & Wang et al. 2020d) \\
\hline Zhao & Retinoblastoma & $(54: 54)$ & qRT-PCR & $p<0.001$ & / & / & / & / & Zhao et al. 2020) \\
\hline Yang & Retinoblastoma & (30: 30$)$ & qRT-PCR & $p<0.05$ & / & / & / & / & Yang et al. 2020) \\
\hline Yao & Retinoblastoma & $(6: 25)$ & qRT-PCR & $p<0.05$ & / & / & / & / & Yao et al. 2020) \\
\hline Zhu & Cervical cancer & $(52: 52)$ & qRT-PCR & $p<0.01$ & $p=0.04$ & / & $p=0.027$ & $p=0.015$ & Zhu et al. 2018a) \\
\hline Chen & Cervical cancer & (30: 30$)$ & qRT-PCR & $p<0.001$ & $p=0.033$ & $p=0.038$ & / & / & Chen et al. 2019b) \\
\hline Liu & Cervical cancer & $(35: 35)$ & qRT-PCR & $p<0.05$ & $p<0.05$ & / & / & $p<0.01$ & Liu et al. 2020) \\
\hline $\mathrm{Xu}$ & Thyroid cancer & $(36: 36)$ & qRT-PCR & $p<0.01$ & $p<0.01$ & $p<0.01$ & I & / & Xu et al. 2018b) \\
\hline Liu & Thyroid cancer & $(77: 77)$ & qRT-PCR & $p<0.01$ & / & / & / & $p=0.025$ & Liu et al. 2018) \\
\hline $\mathrm{Du}$ & Thyroid cancer & $(24: 24)$ & qRT-PCR & $p<0.01$ & / & / & / & / & Du et al. 2021) \\
\hline Song & $\begin{array}{l}\text { Nasopharyngeal } \\
\text { cancer }\end{array}$ & (108: 108) & qRT-PCR & $p<0.05$ & / & / & / & $p=0.0005$ & Song et al. 2016) \\
\hline Cheng & $\begin{array}{l}\text { Nasopharyngeal } \\
\text { cancer }\end{array}$ & $(10: 20)$ & qRT-PCR & $p<0.05$ & / & / & / & / & Cheng et al. 2018) \\
\hline Shi & $\begin{array}{l}\text { Nasopharyngeal } \\
\text { cancer }\end{array}$ & $(40: 40)$ & qRT-PCR & $p<0.05$ & / & / & / & / & Shi et al. 2020) \\
\hline Zhao & $\begin{array}{l}\text { Nasopharyngeal } \\
\text { cancer }\end{array}$ & $(25: 25)$ & qRT-PCR & $p<0.05$ & / & / & / & / & Zhao et al. 2020) \\
\hline Hao & Melanoma & $(15: 15)$ & qRT-PCR & $p<0.01$ & / & / & / & / & Hao et al. 2021) \\
\hline Wang & Leukemia & $(20: 62)$ & qRT-PCR & $p<0.05$ & / & / & / & / & Wang et al. 2020a) \\
\hline
\end{tabular}

\section{XIST in pancreatic cancer}

Due to the poor prognosis, pancreatic cancer has nearly as many deaths $(466,003)$ as new cases $(495,773)$, making pancreatic cancer the 7th leading cause of death for both men and women (Sung et al. 2021). In many countries, morbidity and mortality rates have remained stable or increased slightly, reflecting the growing prevalence of diabetes, obesity, and alcohol consumption. Therefore, further investigation of the pathogenic mechanism underlying pancreatic cancer is needed.

Sun et al. (2018a) reported that XIST was increased in pancreatic cancer tissues and upregulation of XIST promoted cell growth, migration, and invasion of pancreatic cancer. A mechanistic analysis showed that miR-34a-5p was a target of XIST and could rescue the malignant activities mediated by XIST in pancreatic cancer. As a result, a number of investigators focused on the role of XIST in the regulation of pancreatic cancer. It was further demonstrated that XIST promoted pancreatic cancer cell progression through upregulating EGFR (Fig. 1S), iASSP (Fig. 1T), YAP (Fig. 1U), ZEB1 (Fig. 1V), TGF- $\beta 2$ (Fig. $1 \mathrm{~W}$ ), and Notch1 (Fig. 1X) by modulating miR-133a (Wei et al. 2017), miR-140/miR-124 (Liang et al. 2017), miR-34a (Zou et al. 2020), miR-429 (Shen et al. 2019), miR-141-3p (Sun and Zhang 2019), and miR-137 (Liu et al. 2020), respectively. 


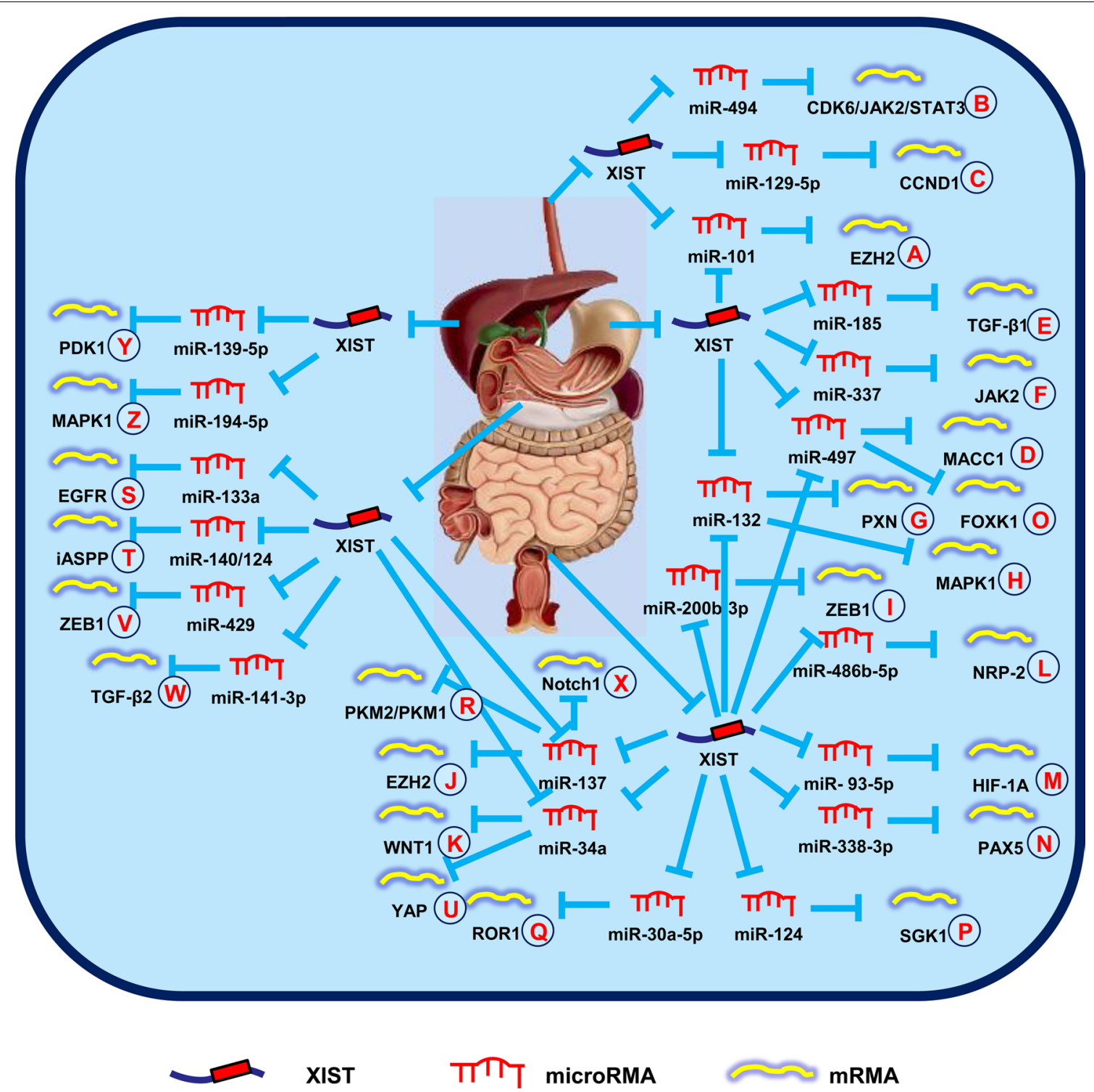

Fig. 1 XIST mediates mechanisms involved in digestive system tumors. A XIST could promote the expression of EZH2 by targeting miR-101. B XIST could promote the expression of CDK6/JAK2/STAT3 by targeting miR-494. C XIST could promote the expression of CCND1 by targeting miR-129-5p. D XIST could promote the expression of MACC1 by targeting miR-497. E XIST could promote the expression of TGF- $\beta 1$ by targeting miR-185. F XIST could promote the expression of JAK2 by targeting miR-337. G XIST could promote the expression of PXN by targeting miR-132. H XIST could promote the expression of MAPK1 by targeting miR-132-3p. I XIST could promote the expression of ZEB1 by targeting miR-200b-3p. J XIST could promote the expression of EZH2 by targeting miR-137. K XIST could promote the expression of WNT1 by targeting miR-34a. L XIST could promote the expression of NRP-2 by targeting miR-486b-5p. M XIST could promote the expression of HIF-1A by targeting miR-93-5p. N XIST could promote the expression of PAX5 by targeting miR-338-3p. O XIST could promote the expression of FOXK1 by targeting miR-497-5p. P XIST could promote the expression of SGK1 by targeting miR-124. Q XIST could promote the expression of ROR1 by targeting miR-30a-5p. R XIST could promote the expression of PKM2/PKM1 by targeting miR-137. S XIST could promote the expression of EGFR by targeting miR-133a. T XIST could promote the expression of iASPP by targeting miR-140/124. U XIST could promote the expression of YAP by targeting miR-34a. V XIST could promote the expression of ZEB1 by targeting miR-429. W XIST could promote the expression of TGF- $\beta 2$ by targeting miR-141-3p. X XIST could promote the expression of Notch1 by targeting miR-137. Y XIST could promote the expression of PDK1 by targeting miR-139-5p. Z XIST could promote the expression of MAPK1 by targeting miR-194-5p

These findings convincingly indicated that XIST provided a diagnostic and therapeutic option for pancreatic cancer.

\section{XIST in hepatocellular carcinoma (HCC)}

Primary liver cancer is the 7 th most common cancer and the 3rd cause of deaths globally, with approximately 
905,677 new cases and 830,180 deaths (Sung et al. 2021). HCC accounts for $75-85 \%$ of primary liver cancers, the main risk factors of which are chronic hepatitis $\mathrm{B}$ or $\mathrm{C}$ virus infection, aflatoxin-contaminated food, excess body weight, heavy alcohol consumption, cigarette smoking, and type 2 diabetes (Sung et al. 2021). Therefore, it is important to identify the diagnostic biomarker and therapeutic target of $\mathrm{HCC}$.

Liu et al. (2019) showed that XIST enhanced cell proliferation by targeting miR-200b-3p, which implied that XIST was a novel therapeutic target in HCC. Another study showed that suppression of XIST inhibited cell growth and promoted cell apoptosis by regulating miR488 expression in HCC (Dong et al. 2020). Recently, the mechanism underlying XIST regulation of the progression of HCC has been shown to involve upregulation of PDK1 (Fig. 1Y) and MAPK1 (Fig. 1Z) by modulating miR-139-5p (Mo et al. 2017) and miR-194-5p (Kong et al. 2018), respectively.

These data demonstrated that XIST functioned as a novel prognostic marker and therapeutic target for HCC.

\section{The role of XIST in respiratory system tumors XIST in laryngeal cancer}

Laryngeal cancer belongs to head and neck tumors based on anatomic site and respiratory system tumors based on function. Laryngeal squamous cell carcinoma (LSCC) is the most common pathologic type of laryngeal cancer. It has been estimated that there will be 12,620 new cases of LSCC and 3770 deaths in the United States in 2021 (Siegel et al. 2021). Therefore, it is important to clarify the pathogenesis underlying laryngeal cancer to develop optimal treatment regimens.

Xiao et al. (2019) showed that XIST expression was highly increased in LSCC. XIST has an important role in the proliferation, invasion, and metastasis of LSCC by regulating the miR-124/EZH2 signals (Fig. 2A). Similar results indicated that XIST promoted carcinogenic cell behavior by regulating the miR-144/IRS1 axis in LSCC, suggesting a potential therapeutic target for LSCC patient treatment (Fig. 2B) (Cui et al. 2020). Furthermore, Liu et al. (2020) reported that XIST upregulated TRIB2 expression in part by sequestration of miR-125b-5p, which in turn expedited LSCC cell progression (Fig. 2C).

In summary, XIST has a key role in the diagnosis and prognosis of LSCC.

\section{XIST in lung cancer}

With an estimated 2,206,771 new cases and 1,796,144 deaths annually, lung cancer ranks 2nd in morbidity and 1st in mortality (Sung et al. 2021). Approximately two-thirds of lung cancer deaths worldwide can be attributed to smoking (Sung et al. 2021). Other factors include occupational exposure and environmental pollution (Turner et al. 2020). Therefore, the identification of new biomarkers and therapeutic approached is important.

A recent study revealed that the levels of HIFA-AS1 and XIST were upregulated in non-small cell lung cancer (NSCLC), and could be used as predictive biomarkers for NSCLC screening (Tantai et al. 2015). Sun et al. (2019) showed that TCF-4 targeting XIST was strongly correlated with lung cancer progression and macrophage polarization. Additionally, Wang et al. (2017b) showed that suppression of XIST inhibited cell growth and invasion, and induced apoptosis through reciprocal inhibition of miR-186-5p, which could be a new therapeutic biomarker in NSCLC. Many studies have since revealed the essential role of the lncRNA-miRNA-mRNA pathway in lung cancer. Specifically, it has been reported that XIST promoted lung cancer cell activities by upregulating BCL-2 (Fig. 2D), iASSP (Fig. 2E), LARP1 (Fig. 2F), Notch1 (Fig. 2G), RING1 (Fig. 2H), SOD2 (Fig. 2I), CDK8 (Fig. 2J), CBLL1 (Fig. 2K), MDM2 (Fig. 2L), and PAX6 (Fig. 2M) via regulation of miR-449a (Zhang et al. 2017b), miR-140 (Tang et al. 2017), miR-374a (Xu et al. 2017), miR-137 (Wang et al. 2018), miR-744 (Wang et al. 2019), miR-335 (Liu et al. 2019), miR-16 (Zhou et al. 2019), miR212-3p (Qiu et al. 2019), miR-363-3p (Rong et al. 2020), and miR-142-5p (Jiang et al. 2020), respectively.

In addition, XIST influences lung cancer chemoresistance. Sun et al. (2017) demonstrated that XIST expression was upregulated in cisplatin-resistant cells. XIST promotes cell growth through BAG-1-mediated chemoresistance by antagonizing let-7i in lung adenocarcinoma (Fig. 2N). Another group confirmed that XIST regulated autophagy through miR-17/ATG7 signals in NSCLC. Downregulation of XIST overcomes chemoresistance by inhibition of autophagy in NSCLC (Fig. 2O) (Sun et al. 2017). In addition, Tian et al. (2019) showed that knockdown of XIST inhibited chemoresistanceassociated cell growth and migration, as well as induced apoptosis in NSCLC. Upregulation of XIST is correlated with cisplatin resistance through the miR-144-3p/MDR1 signal pathway (Fig. 2P). Furthermore, Xu et al. (2020) concluded that suppression of XIST promoted cisplatin (DDP) chemosensitivity by combining with SMAD2 to inhibit NLRP3 and p53 transcription. Therefore, XIST might be used as a key biomarker to predict DDP efficacy in NSCLC. Recently, it was reported that XIST competitively bound to miR-520 in regulating DDP resistance through BAX, and was in turn involved in apoptosis via the p53 signaling pathway (Liu et al. 2021).

In summary, the data implied that XIST had a vital role in lung cancer progression and functioned as a diagnostic and therapeutic target for lung cancer. 


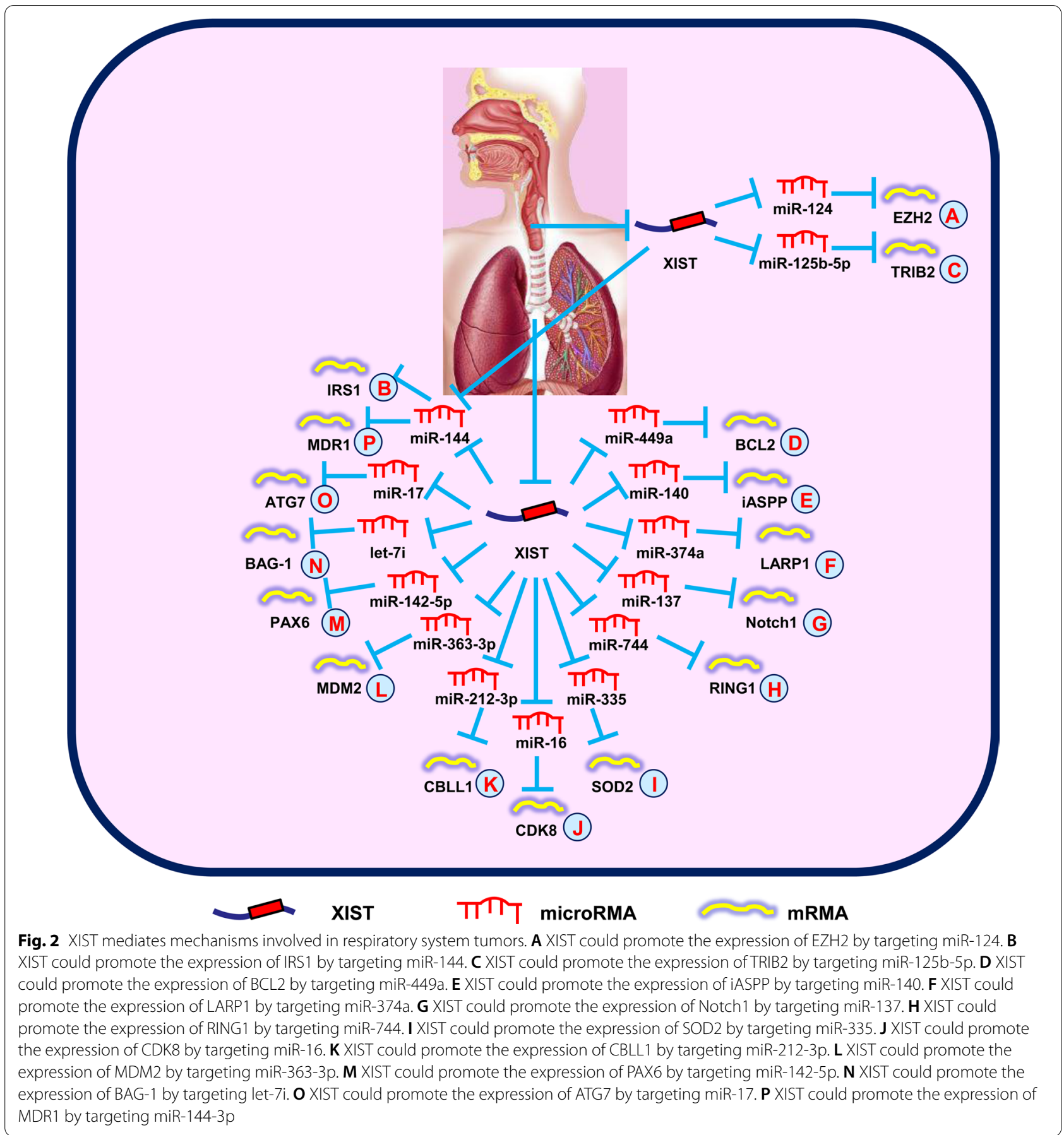

\section{The role of XIST in nervous system tumors XIST in glioma}

Gliomas are the most common invasive nervous system tumors. Despite surgery combined with chemoradiotherapy, the survival of patients with gliomas is approximately 15 months (Thomas et al. 2014). Therefore, it is important to further investigate the gene regulatory networks to improve the treatment of gliomas.
Emerging evidence has shown that XIST expression was elevated in gliomas. Suppression of XIST inhibits tumor progression by reducing cell growth, invasion, and migration, as well as inducing apoptosis, which is mediated by miR-152 in gliomas (Yao et al. 2015). A subsequent study showed that XIST silencing suppressed glioma metastasis and angiogenesis in vivo and in vitro, which was mediated by miR-429 (Cheng et al. 2017). 
Recently, the lncRNA-miRNA-mRNA networks in gliomas have been elucidated. Yu et al. (2017) reported that downregulation of XIST limited cell angiogenesis by regulating FOXC1 and $\mathrm{ZO}-2$ expression, which increased miR-137 expression in gliomas (Fig. 3A). Moreover, Rac1 is a target of the XIST-miR-137 regulatory axis, which is involved in glioma cell proliferation (Fig. 3B) (Wang et al. 2017c). Another study showed that XIST silencing suppressed cell viability, invasion, migration, and glucose metabolism in gliomas. Silencing of XIST decreases tumorigenicity through the IncRNA-XIST/miR-126/IRS1/ PI3K/Akt axis in gliomas (Fig. 3C) (Cheng et al. 2020). Moreover, the XIST/miR-133a/SOX4 axis also promotes cell growth, metastasis, and the epithelial-mesenchymal transition (EMT), providing a novel target for glioma treatment (Fig. 3D) (Luo et al. 2020). Recently, it was reported that downregulation of XIST suppressed cell growth, invasion, and migration, and accelerated apoptosis of glioma cells. Mechanistic investigations showed that XIST, miR-204-5p, and Bcl-2 formed a network to regulate cell progression in gliomas (Fig. 3E) (Shen et al. 2020). Additionally, SRC-1 was shown to elevate glioblastoma stemness by modulating the XIST/miR-152/KLF4 axis, which provided a new diagnostic and therapeutic biomarker for glioblastomas (Fig. 3F) (Gong et al. 2021).

Chemotherapy and radiotherapy also have important roles in the treatment of gliomas. A recent study revealed that XIST enhanced glioma cell chemoresistance to temozolomide via regulating miR-29c/SP1/MGMT axis, which could be a novel target for glioma treatment ( $\mathrm{Du}$ et al. 2017). Another research group reported that XIST expedited cell growth and invasion, and suppressed cell apoptosis through inhibiting the radiosensitivity of gliomas by increasing CREB1 expression via sponging miR329-3p (Fig. 3G) (Wang et al. 2020c).

In summary, these data suggested that XIST played an important role in glioma progression, and functioned as a novel therapeutic target.

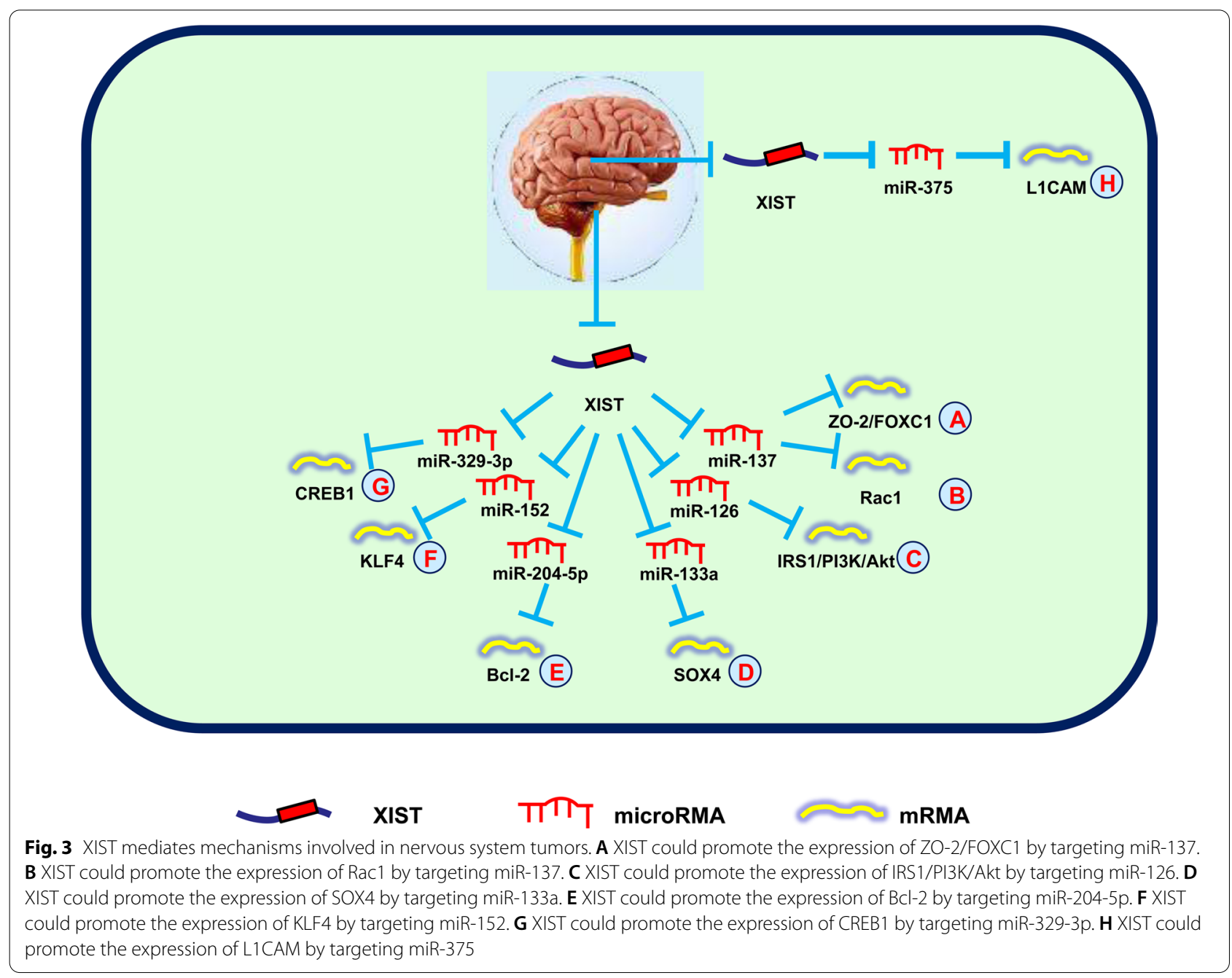




\section{XIST in neuroblastoma (NB)}

NBs consist of undifferentiated neuroblasts, while NBs from different sites have different clinical symptoms (Swift et al. 2018). NBs often occur in children $<5$ years of age and is associated with cellular changes triggered by environmental stimuli; however, only $1-2 \%$ of NB patients have a family history, and the vast majority of NB patients are caused by unknown factors (Swift et al. 2018). Hence, an overall understanding of NB development can facilitate NB surveillance.

Zhang et al. (2019b) demonstrated that XIST decreased DKK1 expression through EZH2, thus accelerating the proliferation, migration, and invasion of NB cells. A subsequent study showed that XIST knockdown limited cell growth and elevated radiosensitivity of NBs by regulating the miR-375/L1CAM signals, thereby confirming that XIST may be a promising biomarker for NBs (Fig. 3H) (Yang et al. 2020).

Taken together, these results indicated that XIST had an oncogenic role in NBs.

\section{Oncogenic role of XIST in other systems XIST in osteosarcoma (OS)}

OSs are a common type of bone tumor in children and young adults that is derived from mesenchymal tissues of bone. OSs account for $15 \%$ of diagnosed malignancies in children and adolescents worldwide and severely affect their health (Aljubran et al. 2009). Epidemiological statistics have shown that when metastasis occurs, survival rates decrease from $65-70 \%$ to $19-30 \%$ (Aljubran et al. 2009). Therefore, identifying novel therapeutic targets and the underlying physiologic mechanism are important for OS treatment.

A study revealed that upregulation of XIST was associated with advanced clinical stage, advanced tumor size, distant metastasis, and poor overall survival rate (Li et al. 2017). Another study reported that XIST promoted cell growth by regulating P21 expression, thus serving as a potential biomarker of OSs (Xu et al. 2017). Similarly, inhibition of XIST suppresses cell growth, migration, and invasion in OSs (Wang et al. 2019). Moreover, knockdown of XIST induces cell apoptosis via modulation of the NF-kB/PUMA axis in OSs (Gao et al. 2019). Li et al. (2019b) reported that XIST promotes cell growth and invasion via suppression of miR-137 expression, thus providing a potential therapeutic target for OSs.

Recently, a series of studies showed that XIST promoted OS cell development by the IncRNA-miRNAmRNA networks. Lv et al. (2018) first reported that XIST was responsible for cell growth and invasion by modulating the miR-320b/RAP2B axis in OSs (Fig. 4A). Another group revealed that XIST functioned as a ceRNA to sequester miR-193a-3p expression, which modulated the target gene, RSF1 (Fig. 4B) (Wu et al. 2017b). In addition, silencing of XIST suppresses cell proliferation and autophagy by inhibiting the AKT/mTOR axis and antagonizing miR-375-3p in OSs (Fig. 4C) (Sun et al. 2019). Shortly thereafter, XIST was shown to promote cell migration, invasion, and EMT by regulating miR-153/ SNAIL signaling, thus serving as a novel therapeutic biomarker for OSs (Fig. 4D) (Wen et al. 2020). Recently, Hai et al. (2021) reported that overexpressed XIST promoted iASPP expression to stimulate cell proliferation by sponging miR-124-3p in OSs (Fig. 4E).

These findings showed that XIST is a promising therapeutic target for inhibiting OS progression.

\section{XIST in bladder cancer (BC)}

BCs are the 10th most common tumors worldwide, with an estimated 573,278 new cases and 212,536 deaths in 2021 (Siegel et al. 2021). BCs are approximately four times more common in men than in women. Although cigarette smoking, exposure to chemical products, chronic infections, aromatic amines, and other pathogenic factors have been identified, the specific pathologic mechanism underlying $\mathrm{BC}$ development is unknown (Siegel et al. 2021). Thus, it is essential to identify new diagnostic biomarkers and the molecular mechanism underlying BCs.

$\mathrm{Xu}$ et al. (2018a) showed that silencing XIST suppressed cell clone formation and EMT in BCs, which was partially restored by miR-200c knockdown. Another group reported that XIST knockdown reduced p53 expression to inhibit cell growth and migration through combination with TET1 in BCs (Hu et al. 2019). Moreover, XIST promotes cell migration and growth by antagonizing miR-133a, suggesting a possible therapeutic marker for BCs (Zhou et al. 2019). The XIST-miRNAmRNA regulatory networks have also been shown to be present in BCs. Xiong et al. (2017) indicated that XIST stimulated cell proliferation, migration, and invasion through miR-124-dependent androgen receptor modulation (Fig. 4F). Another group reported that downregulation of XIST impaired cell proliferation and metastasis by modulating the miR-139-5p-mediated Wnt/ $\beta$-catenin axis in BCs (Fig. 4G) (Hu et al. 2017). Recently, targeting the XIST/miR-335 axis was shown to elevate the antitumor effects of platycodin D in BCs, thus suggesting a therapeutic strategy for BCs (Chen et al. 2020).

Overall, the data indicated that XIST acts as an oncogene in $\mathrm{BC}$ progression.

\section{$X I S T$ in retinoblastoma $(R B)$}

$\mathrm{RBs}$ are the most common intraocular malignancies in infants and young children. RBs are derived from photoreceptor precursor cells and are prone to intracranial and 


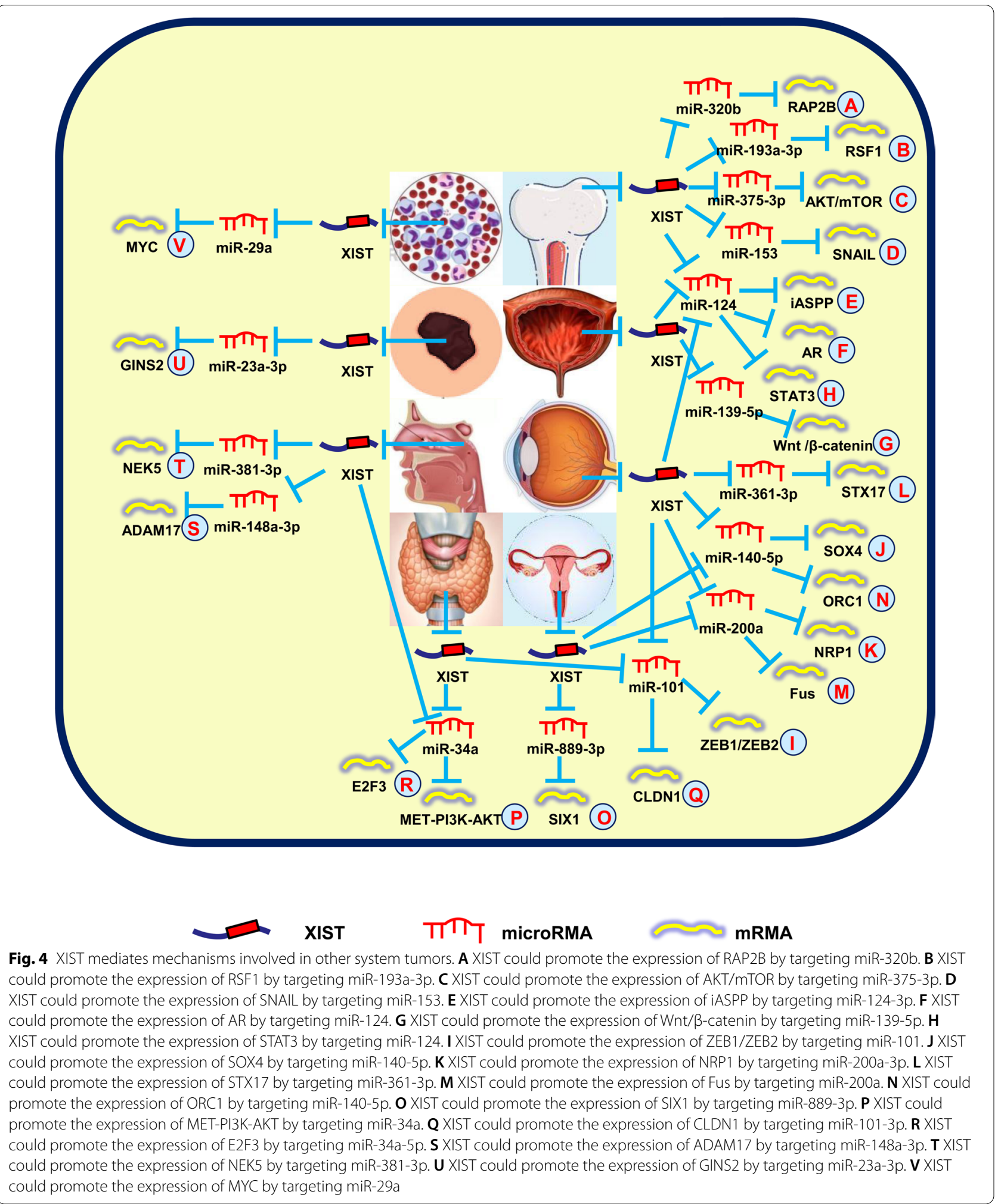

distant metastasis. Patients with early RBs can undergo surgical treatment, or receive radiotherapy, chemotherapy, or other comprehensive measures; however, advanced RBs often lead to a poor prognosis. Therefore, it is necessary to identify effective diagnostic targets and treatment strategies for RBs. 
Lyu et al. (2019) reported that lncRNA NKILA might suppress the growth, invasion, and migration of RBs via XIST knockdown. Upregulation of XIST accelerates cell growth, invasion, and EMT by modulating miR-142-5p expression (Xu and Tian 2020).

Recently, a large number of studies indicated that XIST stimulated RB cell progression via the lncRNA-miRNAmRNA axis. Hu et al. (2018) reported that XIST expedited cell growth by regulating the miR-124/STAT3 axis in RBs (Fig. 4H). In addition, XIST elevates the development of $\mathrm{RB}$ by functioning as a miR-101 sponge to regulate $\mathrm{ZEB} 1 / \mathrm{ZEB} 2$ expression and providing a new therapeutic choice for RBs (Fig. 4I) (Cheng et al. 2019). Another study pointed out that XIST was upregulated in RBs, and suppression of XIST inhibited cell growth and invasion through miR-140-5p/SOX4 signals, suggesting a novel understanding of the mechanism underlying RB (Fig. 4J) (Wang et al. 2020d). Also, XIST silencing increases the PI3K-Akt/MAPK-ERK axis mediated by miR-200a-3p, which affects cell growth, invasion, apoptosis, and EMT in RBs (Fig. 4K) (Zhao et al. 2020). Subsequent studies showed that XIST enhanced the aggressive phenotype of RB cells through miR-361-3p/STX17 signals, functioning as an oncogenic lncRNA (Fig. 4L) (Yang et al. 2020).

XIST is also involved in the chemosensitivity impact on RB patients. Yao et al. (2020) showed that XIST expression was increased in RB tissues. Silencing of XIST inhibits cell development and expedites vincristine sensitivity via functioning as a miR-204-5p sponge, highlighting a potential therapeutic target for RBs.

These findings verified that XIST participates in RB development and functions as a therapeutic and prognostic biomarker.

\section{XIST in cervical cancer (CC)}

$\mathrm{CC}$ is the 4th most frequently diagnosed tumor and the 6th leading cause of cancer death in women, with an estimated 66,570 new cases and 12,940 deaths in the United States in 2021 (Turner et al. 2020). Although tumor screening and HPV vaccination have reduced the incidence of $\mathrm{CC}$, the pathogenesis and treatment warrant further study.

Zhu et al. (2018a) reported that XIST promoted CC progression by regulating the miR-200a/Fus axis while serving as a ceRNA (Fig. 4M). Another group showed that downregulation of XIST inhibited cell growth through miR-140-5p/ORC1 signals in CC (Fig. 4N) (Chen et al. 2019b). Additionally, XIST silencing also impairs cell growth, invasion, and migration by modulating the miR-889-3p/SIX1 signaling pathway and serving as a novel target for the progression of new countermeasures of CC (Fig. 4O) (Liu et al. 2020).
The underlying mechanism of XIST in CC still needs further study.

\section{XIST in thyroid cancer}

The incidence of thyroid cancer is 586,202 cases worldwide, ranking 9th in 2021 (Siegel et al. 2021). Women are three times more likely to have thyroid cancer than men, but the death rate is not high (Siegel et al. 2021). The only identified risk factor for thyroid cancer is ionizing radiation. Therefore, improving thyroid cancer treatment, including medications and surgery, is a pressing problem.

$\mathrm{Xu}$ et al. (2018b) showed that abnormally overexpressed XIST was positively correlated with lymph node metastasis and TNM stage. The molecular mechanism indicated that XIST functions as an oncogene with respect to growth, invasion, and migration by modulating miR-141 in thyroid cancer. A few months later, another research group reported that XIST functioned as a miR34a sponge, competing with MET to regulate cell growth in thyroid cancer (Fig. 4P) (Liu et al. 2018). Furthermore, XIST facilitates cell invasion and migration by directly modulating the miR-101-3p/CLDN1 axis and providing new insight into thyroid cancer treatment (Fig. 4Q) (Du et al. 2021).

Overall, these data indicated that XIST has an oncogenic role in thyroid cancer and represents a new therapeutic target.

\section{XIST in nasopharyngeal carcinoma (NPC)}

NPC is the most common malignant tumor of the oral cavity and pharynx, and squamous cell carcinoma is the most common pathological type. Early stage NPC is easy to be misdiagnosed because of its hidden location and lack of features. Therefore, it is essential to identify an diagnostic marker for early-stage NPC.

A recent study revealed that XIST was upregulated in NPC and led to shorter survival time. An analysis of the mechanism NPC development indicated that XIST promoted NPC cell growth in part by increasing E2F3 through antagonizing miR-34a-5p expression (Fig. 4R) (Song et al. 2016). Another study pointed out that suppression of XIST inhibited cell growth and increased radiosensitivity of NPC cells by upregulating miR-29c expression, offering a novel therapeutic strategy for NPC patients (Han et al. 2017). Cheng et al. (2018) reported that downregulation of XIST induced cell apoptosis and suppressed cell growth and invasion by sponging miR491-5p in NPC. In addition, Shi et al. (Shi et al. 2020) demonstrated that knockdown of XIST suppressed cell proliferation, metastasis, and EMT by regulating the miR-148a-3p/ADAM17 signal pathway in NPC (Fig. 4S). Moreover, Zhao et al. (2020) indicated that downregulation of XIST inhibited cell glycolysis, migration, and 
invasion by modulating the miR-381-3p/NEK5 axis in NPC (Fig. 4T).

These data indicate that XIST might be used as a prognostic biomarker and therapeutic target for NPC.

\section{XIST in melanoma}

The estimated incidence of melanomas in 2021 is the 5th highest among malignancies in both men and women (Siegel et al. 2021). Excessive ultraviolet exposure is thought to be the most important reason for the sharp rise in melanoma rates, which accounts for approximately $90 \%$ of skin cancer deaths. Therefore, it is important to explore early diagnostic and therapeutic biomarkers for melanomas.

Hao et al. (2021) revealed that XIST facilitated oncogenic behavior of melanomas by sequestering miR23a-3p and indirectly targeting its downstream gene, GINS2, thus providing a potential target (Fig. 4U).

This result shows that XIST serves as an oncogene to accelerate melanoma progression.

\section{XIST in leukemia}

Leukemia has the sixth (male) and eighth (female) highest mortality rate among malignancies, but morbidity ranks 9th (male) and 10th (female). The causes of leukemia are not fully understood. The mutations of some genes in hematopoietic cells caused by various causes lead to the formation of clonal abnormal hematopoietic cells. Therefore, it is imperative to identify therapeutic targets for leukemia.

Wang et al. (2020a) reported that XIST was increased in acute myeloid leukemia (AML) cells. Functional assays demonstrated that XIST knockdown decreased the expression of MYC by antagonizing miR-29a, thereby suppressing viability, reducing chemoresistance, and promoting apoptosis of AML cells (Fig. 4V).

In summary, this study offers new insights into the mechanism by which XIST regulates leukemia.

\section{Conclusion and future perspectives}

Cancer refers to a tumor formed by abnormal proliferation of cells in the local tissue under the stimulation of various tumorigenic factors. Malignant tumors can destroy the structure and function of tissues and organs, causing necrosis and hemorrhage combined with infection, and the patient may eventually die due to organ failure. The Union for International Cancer Control has put forward the concept of tertiary prevention of malignant tumors. The secondary prevention of tumors is how to detect cancer at an early stage and treat it promptly. Therefore, many researchers have carried out studies on early diagnostic biomarkers and therapeutic targets of tumors.
LncRNAs have been demonstrated to be abnormally expressed in many diseases, including cancers, functioning as oncogenes or tumor suppressors. Recently, it has been repeatedly shown that XIST was overexpressed in various tumors, acting as an oncogenic lncRNA (Wu et al. 2017a; Chen et al. 2016; Zhang et al. 2019a). The overexpression of XIST is related to the onset and development of tumors, such as cell viability, autophagy, clone formation, angiogenesis, proliferation, migration, invasion, apoptosis, EMT, metastasis, tumor glycolysis, cell cycle regulation, radiosensitivity, chemoresistance, and stem cell formation. In contrast, XIST knockdown significantly inhibits the aggressive phenotypes of those tumors. Therefore, XIST is considered to be a potential biomarker and therapeutic target of tumors; however, the mechanistic investigations of XIST need to be further explored.

In our review, we summarized the carcinogenesis of XIST in multiple tumors. First, XIST promotes tumor development and prognosis by interacting with miRNAs and/or targeting proteins. The overexpression of XIST is closely related to the clinicopathologic features of tumors. Second, XIST competitively binds to mRNAs by adsorbing miRNAs, which constitutes a regulatory model of the "XIST-miRNAs-mRNAs" axis. Finally, XIST plays an important role in the sensitivity of tumor radiotherapy and chemotherapy, which can be targeted to improve the efficacy of tumor radiotherapy and chemotherapy.

It should be noticed that there existed some limitations in our manuscript. First, XIST was overexpressed in many non-sex-related human cancers and downregulated in some sex-specific cancers, such as breast cancer, ovarian cancer, and prostate cancer (Zhang et al. 2020; Guo et al. 2021; Du et al. 2017). Whether this phenomenon is related to the fact that XIST is a key regulator of mammalian XCI deserves further study. Another reason may be due to the differences in the source of tumor tissue and extracellular microenvironment. Second, in the same type of tumor, such as HCC, OS, and renal cell carcinoma, the expression of XIST was also inconsistent (Lin et al. 2018; Zhang and Xia 2017; Sun et al. 2019). These inconsistencies could be due to sex differences between samples, or XIST controls cancer development at multiple levels. Finally, there were not enough data to fully confirm the relationship between XIST and clinicopathological features. Therefore, larger and multicenter studies are needed to determine this correlation.

In all, our review demonstrated that XIST is overexpressed in most human tumors and involved in multiple layers of carcinogenesis. The effects of XIST on tumors are complex, including many tumorigenic regulatory networks and tumor-associated miRNAs. Therefore, a sufficient understanding of XIST in molecular biology will 
be helpful for the utilization of XIST as a diagnostic biomarker and therapeutic target for clinical cancer therapy.

\begin{abstract}
Abbreviations
LncRNA: Long non-coding RNA; XIST: X-inactive specific transcript; XCl: X-chromosome inactivation; EC: Esophageal cancer; GC: Gastric cancer; CRC : Colorectal cancer; HCC: Hepatocellular carcinoma; NB: Neuroblastoma; OS: Osteosarcoma; BC: Bladder cancer; RB: Retinoblastoma; CC: Cervical cancer; NPC: Nasopharyngeal carcinoma; ESCC: Esophageal squamous cell carcinoma; 5FU: 5-Fluorouracil; LSCC: Laryngeal squamous cell carcinoma; NSCLC: Nonsmall cell lung cancer; DDP: Cisplatin; EMT: Epithelial-mesenchymal transition; AML: Acute myeloid leukemia.
\end{abstract}

\section{Acknowledgements}

We thank Yun Cui from Department of Urology, Beijing Chaoyang Hospital for helping us preparing the manuscript.

\section{Authors' contributions}

KW, JY and MQ conceived the review; KW, XF and XW wrote the manuscript. All authors read and approved the final manuscript.

\section{Funding}

This work was supported by National Natural Science Foundation of China (Grant No. 82072835) to K Wang, Key Research and Development Joint Program of Liaoning Province (Grant No. 2020JH 2/10300139) to KWang, Natural Science Foundation of Liaoning Province (Grant No. 2019-MS-360) to KWang, Shenyang Science and Technology Bureau Plan Projects (Grant No. 20-2054-076) to K Wang, 345 Talent Project of Shengjing Hospital of China Medical University to KWang and Natural Science Foundation of Liaoning Province (Grant No. 2019-MS-371) to M Qi.

\section{Availability of data and materials}

Not applicable.

\section{Declarations}

Ethics approval and consent to participate

Not applicable.

\section{Consent for publication}

Not applicable.

\section{Competing interests}

The authors declare that they have no competing interests.

\section{Author details}

1'Department of Gastroenterology, Shengjing Hospital of China Medical University, Shenyang 110004, China. ${ }^{2}$ Department of Clinical Genetics, Shengjing Hospital of China Medical University, Shenyang 110004, China. ${ }^{3}$ Department of Urology, Shengjing Hospital of China Medical University, \#36 Sanhao Street, Heping, Liaoning 110004 Shenyang, China.

Received: 11 May 2021 Accepted: 6 December 2021

Published online: 20 December 2021

\section{References}

Aljubran AH, Griffin A, Pintilie M, et al. Osteosarcoma in adolescents and adults: survival analysis with and without lung metastases. Ann Oncol. 2009;20(6):1136-41.

Brown CJ, Ballabio A, Rupert JL, et al. A gene from the region of the human $X$ inactivation centre is expressed exclusively from the inactive $X$ chromosome. Nature. 1991;349(6304):38-44.

Chen D, Ju H, Lu Y, et al. Long non-coding RNA XIST regulates gastric cancer progression by acting as a molecular sponge of miR-101 to modulate EZH2 expression. J Exp Clin Cancer Res. 2016;35(1):142.
Chen D, Chen L, Lu Y, et al. Long noncoding RNA XIST expedites metastasis and modulates epithelial-mesenchymal transition in colorectal cancer. Cell Death Dis. 2017;8(8): e3011.

Chen Z, Hu X, Wu Y, et al. Long non-coding RNA XIST promotes the development of esophageal cancer by sponging miR-494 to regulate CDK6 expression. Biomed Pharmacother. 2019a;109:2228-36.

Chen X, Xiong D, Ye L, et al. Up-regulated IncRNA XIST contributes to progression of cervical cancer via regulating miR-140-5p and ORC1. Cancer Cell Int. 2019b;19:45.

Chen D, Chen T, Guo Y, et al. Platycodin D (PD) regulates LncRNA-XIST/miR-335 axis to slow down bladder cancer progression in vitro and in vivo. Exp Cell Res. 2020;396(1): 112281.

Cheng Z, Li Z, Ma K, et al. Long non-coding RNA XIST promotes glioma tumorigenicity and angiogenesis by acting as a molecular sponge of miR-429. J Cancer. 2017;8(19):4106-16.

Cheng Q, Xu X, Jiang H, et al. Knockdown of long non-coding RNA XIST suppresses nasopharyngeal carcinoma progression by activating miR491-5p. J Cell Biochem. 2018;119(5):3936-44.

Cheng Y, Chang Q, Zheng B, et al. LncRNA XIST promotes the epithelial to mesenchymal transition of retinoblastoma via sponging miR-101. Eur J Pharmacol. 2019;843:210-6.

Cheng Z, Luo C, Guo Z. LncRNA-XIST/microRNA-126 sponge mediates cell proliferation and glucose metabolism through the IRS1/PI3K/Akt pathway in glioma. J Cell Biochem. 2020;121(3):2170-83.

Cui C, Li Y, Cui X, et al. IncRNA XIST promotes the progression of laryngeal squamous cell carcinoma by sponging miR-144 to regulate IRS1 expression. Oncol Rep. 2020;43(2):525-35.

Dong Z, Yang J, Zheng F, et al. The expression of IncRNA XIST in hepatocellular carcinoma cells and its effect on biological function. J BUON. 2020;25(5):2430-7.

Du Y, Weng X, Wang L, et al. LncRNA XIST acts as a tumor suppressor in prostate cancer through sponging miR-23a to modulate RKIP expression. Oncotarget. 2017:8(55):94358-70.

Du P, Zhao H, Peng R, et al. LncRNA-XIST interacts with miR-29c to modulate the chemoresistance of glioma cell to TMZ through DNA mismatch repair pathway. Biosci Rep. 2017;37(5): BSR20170696.

Du Y, Liang Y, Cao Y, et al. LncRNA XIST promotes migration and invasion of papillary thyroid cancer cell by modulating MiR-101-3p/CLDN1 axis. Biochem Genet. 2021;59(2):437-52.

Fang J, Sun C, Gong C. Long noncoding RNA XIST acts as an oncogene in nonsmall cell lung cancer by epigenetically repressing KLF2 expression. Biochem Biophys Res Commun. 2016;478(2):811-7.

Gao W, Gao J, Chen L, et al. Targeting XIST induced apoptosis of human osteosarcoma cells by activation of NF-kB/PUMA signal. Bioengineered. 2019;10(1):261-70.

Gong M, Wang X, Mu L, et al. Steroid receptor coactivator-1 enhances the stemness of glioblastoma by activating long noncoding RNA XIST/miR152/KLF4 pathway. Cancer Sci. 2021;112(2):604-18.

Guo T, Yuan D, Zhang W, et al. Upregulation of long noncoding RNA XIST has anticancer effects on ovarian cancer through sponging miR-106a. Hum Cell. 2021;34(2):579-87.

Hai B, Pan X, Du C, et al. LncRNA XIST promotes growth of human chordoma cells by regulating miR-124-3p/iASPP pathway. Cancers (basel). 2021;13(6):1430.

Han Q, Li L, Liang H, et al. Downregulation of IncRNA X inactive specific transcript (XIST) suppresses cell proliferation and enhances radiosensitivity by upregulating mir-29c in nasopharyngeal carcinoma cells. Med Sci Monit. 2017;23:4798-807.

Hao Y, Liu K, Zhang X, et al. GINS2 was regulated by IncRNA XIST/miR-23a-3p to mediate proliferation and apoptosis in A375 cells. Mol Cell Biochem. 2021;476(3):1455-65.

Hu Y, Deng C, Zhang H, et al. Long non-coding RNA XIST promotes cell growth and metastasis through regulating miR-139-5p mediated Wnt/ $\beta$-catenin signaling pathway in bladder cancer. Oncotarget. 2017;8(55):94554-68.

Hu C, Liu S, Han M, et al. Knockdown of IncRNA XIST inhibits retinoblastoma progression by modulating the miR-124/STAT3 axis. Biomed Pharmacother. 2018;107:547-54.

Hu B, Shi G, Li Q, et al. Long noncoding RNA XIST participates in bladder cancer by downregulating p53 via binding to TET1. J Cell Biochem. 2019;120(4):6330-8. 
Jiang Q, Xing W, Cheng J, et al. Knockdown of IncRNA XIST suppresses cell tumorigenicity in human non-small cell lung cancer by regulating miR142-5p/PAX6 axis. Onco Targets Therapy. 2020;13:4919-29.

Kong Q, Zhang S, Liang C, et al. LncRNA XIST functions as a molecular sponge of miR-194-5p to regulate MAPK1 expression in hepatocellular carcinoma cell. J Cell Biochem. 2018;119(6):4458-68.

Li G, Wu Y, Li Y, et al. High expression of long non-coding RNA XIST in osteosarcoma is associated with cell proliferation and poor prognosis. Eur Rev Med Pharmacol Sci. 2017;21(12):2829-34

Li Y, Zhang Q, Tang X. Long non-coding RNA XIST contributes into drug resistance of gastric cancer cell. Minerva Med. 2019a;110(3):270-2.

$\mathrm{Li} \mathrm{H,} \mathrm{Cui} \mathrm{J,} \mathrm{Xu} \mathrm{B,} \mathrm{et} \mathrm{al.} \mathrm{Long} \mathrm{non-coding} \mathrm{RNA} \mathrm{XIST} \mathrm{serves} \mathrm{an} \mathrm{oncogenic} \mathrm{role} \mathrm{in}$ osteosarcoma by sponging miR-137. Exp Ther Med. 2019b;17(1):730-8.

Li P, Wang L, Li P, et al. Silencing of long non-coding RNA XIST represses gastric cancer progression through blocking NFKB pathway via inhibiting HNF4A-mediated transcription of EPHA1. Cancer Gene Ther. 2020. https://doi.org/10.1038/s41417-020-00220-5.

Li Q, Sun Q, Zhu B. LnCRNA XIST inhibits the progression of oral squamous cell carcinoma via sponging miR-455-3p/BTG2 axis. Onco Targets Therapy. 2020a:13:11211-20.

Li P, Wang L, Li P, et al. Silencing IncRNA XIST exhibits antiproliferative and proapoptotic effects on gastric cancer cells by up-regulating microRNA-132 and down-regulating PXN. Aging (albany NY). 2020b;5:12.

Li W, He Y, Cheng Z. Long noncoding RNA XIST knockdown suppresses the growth of colorectal cancer cells via regulating microRNA-338-3p/PAX5 axis. Eur J Cancer Prev. 2021;30(2):132-42.

Liang S, Gong X, Zhang G, et al. The IncRNA XIST interacts with miR-140/miR124/iASPP axis to promote pancreatic carcinoma growth. Oncotarget. 2017:8(69):113701-18.

Lin X, Huang Z, Chen X, et al. XIST induced by JPX suppresses hepatocellular carcinoma by sponging miR-155-5p. Yonsei Med J. 2018;59(7):816-26.

Liu W, Xu Q. Long non-coding RNA XIST promotes hepatocellular carcinoma progression by sponging miR-200b-3p. Eur Rev Med Pharmacol Sci. 2019;23(22):9857-62.

Liu H, Deng H, Zhao Y, et al. LncRNA XIST/miR-34a axis modulates the cell proliferation and tumor growth of thyroid cancer through MET-PI3KAKT signaling. J Exp Clin Cancer Res. 2018;37(1):279.

Liu X, Cui L, Hua D. Long noncoding RNA XIST regulates miR-137-EZH2 axis to promote tumor metastasis in colorectal cancer. Oncol Res. 2018;27(1):99-106.

Liu A, Liu L, Lu H. LnCRNA XIST facilitates proliferation and epithelialmesenchymal transition of colorectal cancer cells through targeting miR-486-5p and promoting neuropilin-2. J Cell Physiol. 2019. https:// doi.org/10.1002/jcp.28054

Liu J, Yao L, Zhang M, et al. Downregulation of LncRNA-XIST inhibited development of non-small cell lung cancer by activating miR-335/SOD2/ROS signal pathway mediated pyroptotic cell death. Aging (albany NY). 2019;11(18):7830-46.

Liu X, Xie S, Zhang J, et al. Long noncoding RNA XIST contributes to cervical cancer development through targeting miR-889-3p/SIX1 axis. Cancer Biother Radiopharm. 2020;35(9):640-9.

Liu P, Pan Y, Wang D, et al. Long non-coding RNA XIST promotes cell proliferation of pancreatic cancer through miR-137 and Notch1 pathway. Eur Rev Med Pharmacol Sci. 2020;24(23):12161-70.

Liu C, Lu Z, Liu H, et al. LnCRNA XIST promotes the progression of laryngeal squamous cell carcinoma via sponging miR-125b-5p to modulate TRIB2. Biosci Rep. 2020;40(4): BSR20193172.

Liu T, Li R, Liu X, et al. LncRNA XIST acts as a MicroRNA-520 sponge to regulate the Cisplatin resistance in NSCLC cells by mediating BAX through CeRNA network. Int J Med Sci. 2021;18(2):419-31.

Loda A, Heard E. Xist RNA in action: past, present, and future. PLoS Genet. 2019:15(9): e1008333.

Luo C, Quan Z, Zhong B, et al. IncRNA XIST promotes glioma proliferation and metastasis through miR-133a/SOX4. Exp Ther Med. 2020;19(3):1641-8.

LV G, Miao J, Zhang X. Long noncoding RNA XIST promotes osteosarcoma progression by targeting Ras-related protein RAP2B via miR-320b. Oncol Res. 2018;26(6):837-46.

Lyon MF. X-chromosome inactivation and developmental patterns in mammals. Biol Rev Camb Philos Soc. 1972;47(1):1-35.

Lyu X, Ma Y, Wu F, et al. LncRNA NKILA inhibits retinoblastoma by downregulating IncRNA XIST. Curr Eye Res. 2019;44(9):975-9.
Ma L, Zhou Y, Luo X, et al. Long non-coding RNA XIST promotes cell growth and invasion through regulating miR-497/MACC1 axis in gastric cancer. Oncotarget. 2017;8(3):4125-35.

Ma S, Wang Y, Li X, et al. LncRNA XIST promotes proliferation and cisplatin resistance of oral squamous cell carcinoma by downregulating miR27b-3p. J Biol Regul Homeost Agents. 2020;34(6):1993-2001.

Mo Y, Lu Y, Wang P, et al. Long non-coding RNA XIST promotes cell growth by regulating miR-139-5p/PDK1/AKT axis in hepatocellular carcinoma. Tumour Biol. 2017:39(2):1010428317690999.

Parodi S. Xist noncoding RNA could act as a tumor suppressor gene in patients with classical Hodgkin's disease. J Cancer Res Ther. 2020;16(1):7-12.

Qiu H, Yang K, Yu H, et al. Downregulation of long non-coding RNA XIST inhibits cell proliferation, migration, invasion and EMT by regulating miR-212-3p/CBLL1 axis in non-small cell lung cancer cells. Eur Rev Med Pharmacol Sci. 2019;23(19):8391-402.

Rafiee A, Riazi-Rad F, Havaskary M, et al. Long noncoding RNAs: regulation, function and cancer. Biotechnol Genet Eng Rev. 2018;34(2):153-80.

Rong $\mathrm{H}$, Chen B, Wei X, et al. Long non-coding RNA XIST expedites lung adenocarcinoma progression through upregulating MDM2 expression via binding to miR-363-3p. Thorac Cancer. 2020;11(3):659-71.

Shen J, Hong L, Yu D, et al. LncRNA XIST promotes pancreatic cancer migration, invasion and EMT by sponging miR-429 to modulate ZEB1 expression. Int J Biochem Cell Biol. 2019;113:17-26.

Shen J, Xiong J, Shao X, et al. Knockdown of the long noncoding RNA XIST suppresses glioma progression by upregulating miR-204-5p. J Cancer. 2020;11(15):4550-9.

Shi J, Tan S, Song L, et al. LncRNA XIST knockdown suppresses the malignancy of human nasopharyngeal carcinoma through XIST/miRNA-148a-3p/ ADAM17 pathway in vitro and in vivo. Biomed Pharmacother. 2020;121: 109620.

Siegel RL, Miller KD, Sauer AG, et al. Colorectal cancer statistics, 2020. CA Cancer J Clin. 2020;70(3):145-64.

Siegel RL, Miller KD, Fuchs HE, et al. Cancer statistics, 2021. CA Cancer J Clin. 2021;71(1):7-33.

Song P, Ye L, Zhang C, et al. Long non-coding RNA XIST exerts oncogenic functions in human nasopharyngeal carcinoma by targeting miR-34a-5p. Gene. 2016;592(1):8-14.

Song $\mathrm{H}$, He P, Shao T, et al. Long non-coding RNA XIST functions as an oncogene in human colorectal cancer by targeting miR-132-3p. J BUON. 2017;22(3):696-703.

Spizzo R, Almeida MI, Colombatti A, et al. Long non-coding RNAs and cancer: a new frontier of translational research? Oncogene. 2012;31(43):4577-87.

Sun Y, Xu J. TCF-4 regulated IncRNA-XIST promotes M2 Polarization of macrophages and is associated with lung cancer. Onco Targets Therapy. 2019;12:8055-62

Sun J, Zhang Y. LnCRNA XIST enhanced TGF- $\beta 2$ expression by targeting miR-141-3p to promote pancreatic cancer cells invasion. Biosci Rep. 2019;39(7): BSR20190332.

Sun W, Zu Y, Fu X, et al. Knockdown of IncRNA-XIST enhances the chemosensitivity of NSCLC cells via suppression of autophagy. Oncol Rep. 2017;38(6):3347-54

Sun J, Pan L, Chen L, et al. LncRNA XIST promotes human lung adenocarcinoma cells to cisplatin resistance via let-7i/BAG-1 axis. Cell Cycle. 2017;16(21):2100-7.

Sun Z, Zhang B, Cui T. Long non-coding RNA XIST exerts oncogenic functions in pancreatic cancer via miR-34a-5p. Oncol Rep. 2018a;39(4):1591-600.

Sun N, Zhang G, Liu Y. Long non-coding RNA XIST sponges miR-34a to promotes colon cancer progression via Wnt/ $\beta$-catenin signaling pathway. Gene. 2018b;665:141-8

Sun X, Wei B, Peng Z, et al. Knockdown of IncRNA XIST suppresses osteosarcoma progression by inactivating AKT/mTOR signaling pathway by sponging miR-375-3p. Int J Clin Exp Pathol. 2019;12(5):1507-17.

Sun K, Jia Z, Duan R, et al. Long non-coding RNA XIST regulates miR-106b-5p/ P21 axis to suppress tumor progression in renal cell carcinoma. Biochem Biophys Res Commun. 2019;510(3):416-20.

Sung H, Ferlay J, Siegel R, et al. Global cancer statistics 2020: GLOBOCAN estimates of incidence and mortality worldwide for 36 cancers in 185 countries. CA Cancer J Clin. 2021. https://doi.org/10.3322/caac.21660.

Swift CC, Eklund MJ, Kraveka JM, et al. Updates in diagnosis, management, and treatment of neuroblastoma. Radiographics. 2018;38(2):566-80. 
Tang Y, He R, An J, et al. IncRNA XIST interacts with miR-140 to modulate lung cancer growth by targeting iASPP. Oncol Rep. 2017;38(2):941-8.

Tantai J, Hu D, Yang Y, et al. Combined identification of long non-coding RNA XIST and HIF1A-AS1 in serum as an effective screening for non-small cell lung cancer. Int J Clin Exp Pathol. 2015;8(7):7887-95.

Thomas AA, Brennan CW, DeAngelis LM, et al. Emerging therapies for glioblastoma. JAMA Neurol. 2014;71(11):1437-44.

Tian L, Wu Y, Wang D, et al. Upregulation of long noncoding RNA (IncRNA) $X$-inactive specific transcript (XIST) is associated with cisplatin resistance in non-small cell lung cancer (NSCLC) by downregulating microRNA-144-3p. Med Sci Monit. 2019;25:8095-104.

Turner MC, Andersen ZJ, Baccarelli A, et al. Outdoor air pollution and cancer: an overview of the current evidence and public health recommendations. CA Cancer J Clin. 2020;70:460-79.

Wang $\mathrm{K}$, Jin W, Song Y, et al. LncRNA RP1 1-436H11.5, functioning as a competitive endogenous RNA, upregulates BCL-W expression by sponging miR-335-5p and promotes proliferation and invasion in renal cell carcinoma. Mol Cancer. 2017;16(1):166.

Wang H, Shen Q, Zhang X, et al. The long non-coding RNA XIST controls non-small cell lung cancer proliferation and invasion by modulating miR-186-5p. Cell Physiol Biochem. 2017b;41 (6):2221-9.

Wang Z, Yuan J, Li L, et al. Long non-coding RNA XIST exerts oncogenic functions in human glioma by targeting miR-137. Am J Transl Res. 2017c;9(4):1845-55.

Wang X, Zhang G, Cheng Z, et al. Knockdown of LncRNA-XIST suppresses proliferation and TGF- $\beta 1$-induced EMT in NSCLC through the Notch-1 pathway by regulation of miR-137. Genet Test Mol Biomark. 2018;22(6):333-42.

Wang J, Cai H, Dai Z, et al. Down-regulation of IncRNA XIST inhibits cell proliferation via regulating miR-744/RING1 axis in non-small cell lung cancer. Clin Sci (lond). 2019;133(14):1567-79.

Wang W, Shen H, Cao G, et al. Long non-coding RNA XIST predicts poor prognosis and promotes malignant phenotypes in osteosarcoma. Oncol Lett. 2019;17(1):256-62.

Wang C, Li L, Li M, et al. Silencing long non-coding RNA XIST suppresses drug resistance in acute myeloid leukemia through down-regulation of MYC by elevating microRNA-29a expression. Mol Med. 2020a;26(1):114.

Wang N, He J, Jia G, et al. The IncRNA XIST promotes colorectal cancer cell growth through regulating the miR-497-5p/FOXK1 axis. Cancer Cell Int. 2020b;20(1):553.

Wang Y, Li H, Chen J, et al. Overexpression of XIST facilitates cell proliferation, invasion and suppresses cell apoptosis by reducing radio-sensitivity of glioma cells via miR-329-3p/CREB1 axis. Eur Rev Med Pharmacol Sci. 2020c;24(6):3190-203.

Wang Y, Sun D, Sheng Y, et al. XIST promotes cell proliferation and invasion by regulating miR-140-5p and SOX4 in retinoblastoma. World I Surg Oncol. 2020d;18(1):49.

Wang $\mathrm{H}, \mathrm{Li}$ H, Yu Y, et al. Long non-coding RNA XIST promotes the progression of esophageal squamous cell carcinoma through sponging miR-129-5p and upregulating CCND1 expression. Cell Cycle. 2021;20(1):39-53.

Wei W, Liu Y, Lu Y, et al. LncRNA XIST promotes pancreatic cancer proliferation through miR-133a/EGFR. J Cell Biochem. 2017;118(10):3349-58.

Wen J, Jiang Y, Li C, et al. LncRNA-XIST promotes the oxidative stress-induced migration, invasion, and epithelial-to-mesenchymal transition of osteosarcoma cancer cells through miR-153-SNAl1 axis. Cell Biol Int. 2020:44(10):1991-2001.

Wu X, Dinglin X, Wang X, et al. Long noncoding RNA XIST promotes malignancies of esophageal squamous cell carcinoma via regulation of miR-101/ EZH2. Oncotarget. 2017a;8(44):76015-28.

Wu D, Nie X, Ma C, et al. RSF1 functions as an oncogene in osteosarcoma and is regulated by XIST/miR-193a-3p axis. Biomed Pharmacother. 2017b;95:207-14.

Xiao Y, Yurievich U, Yosypovych S. Long noncoding RNA XIST is a prognostic factor in colorectal cancer and inhibits 5-fluorouracil-induced cell cytotoxicity through promoting thymidylate synthase expression. Oncotarget. 2017:8(47):83171-82.

Xiao D, Cui X, Wang X. Long noncoding RNA XIST increases the aggressiveness of laryngeal squamous cell carcinoma by regulating miR-124-3p/EZH2. Exp Cell Res. 2019;381(2):172-8.

Xiong $Y$, Wang L, Li Y, et al. The long non-coding RNA XIST interacted with MiR-124 to modulate bladder cancer growth, invasion and migration by targeting androgen receptor (AR). Cell Physiol Biochem. 2017:43(1):405-18.

Xu C, Tian L. LncRNA XIST promotes proliferation and epithelial-mesenchymal transition of retinoblastoma cells through sponge action of miR-142-5p. Eur Rev Med Pharmacol Sci. 2020;24(18):9256-64.

XuT, Jiang W, Fan L, et al. Upregulation of long noncoding RNA Xist promotes proliferation of osteosarcoma by epigenetic silencing of P21. Oncotarget. 2017;8(60):101406-17.

Xu Z, Xu J, Lu H, et al. LARP1 is regulated by the XIST/miR-374a axis and functions as an oncogene in non-small cell lung carcinoma. Oncol Rep. 2017;38(6):3659-67.

Xu R, Zhu X, Chen F, et al. LncRNA XIST/miR-200c regulates the stemness properties and tumourigenicity of human bladder cancer stem cell-like cells. Cancer Cell Int. 2018a;18:41.

Xu Y, Wang J, Wang J. Long noncoding RNA XIST promotes proliferation and invasion by targeting miR-141 in papillary thyroid carcinoma. Onco Targets Therapy. 2018b;11:5035-43.

Xu X, Zhou X, Chen Z, et al. Silencing of IncRNA XIST inhibits non-small cell lung cancer growth and promotes chemosensitivity to cisplatin. Aging (albany NY). 2020;12(6):4711-26.

Yang L, Li Q, Zhang X, et al. Long non-coding RNA XIST confers aggressive progression via miR-361-3p/STX17 in retinoblastoma cells. Eur Rev Med Pharmacol Sci. 2020;24(20):10433-44.

Yang X, Zhang S, He C, et al. METTL14 suppresses proliferation and metastasis of colorectal cancer by down-regulating oncogenic long non-coding RNA XIST. Mol Cancer. 2020;19(1):46.

Yang L, Cao M, Zhang J, et al. LncRNA XIST modulates HIF-1A/AXL signaling pathway by inhibiting miR-93-5p in colorectal cancer. Mol Genet Genomic Med. 2020;8(4): e1112.

Yang H, Zhang X, Zhao Y, et al. Downregulation of IncRNA XIST represses tumor growth and boosts radiosensitivity of neuroblastoma via modulation of the miR-375/L1CAM axis. Neurochem Res. 2020:45(11):2679-90.

Yao Y, Ma J, Xue Y, et al. Knockdown of long non-coding RNA XIST exerts tumor-suppressive functions in human glioblastoma stem cells by upregulating miR-152. Cancer Lett. 2015;359(1):75-86.

Yao L, Yang L, Song H, et al. Silencing of IncRNA XIST suppresses proliferation and autophagy and enhances vincristine sensitivity in retinoblastoma cells by sponging miR-204-5p. Eur Rev Med Pharmacol Sci. 2020;24(7):3526-37.

Yu H, Xue Y, Wang P, et al. Knockdown of long non-coding RNA XIST increases blood-tumor barrier permeability and inhibits glioma angiogenesis by targeting miR-137. Oncogenesis. 2017;6(3): e303.

Yu J, Dong W, Liang J. Extracellular vesicle-transported long non-coding RNA (LncRNA) X inactive-specific transcript (XIST) in serum is a potential novel biomarker for colorectal cancer diagnosis. Med Sci Monit. 2020;26: e924448.

Yue M, Richard J, Yamada N, et al. Quick fluorescent in situ hybridization protocol for Xist RNA combined with immunofluorescence of histone modification in X-chromosome inactivation. J vis Exp. 2014;26(93): e52053.

Zhang R, Xia T. Long non-coding RNA XIST regulates PDCD4 expression by interacting with miR-21-5p and inhibits osteosarcoma cell growth and metastasis. Int J Oncol. 2017:51(5):1460-70.

Zhang J, Cao Z, Ding X, et al. The IncRNA XIST regulates the tumorigenicity of renal cell carcinoma cells via the miR-302c/SDC1 axis. Int J Clin Exp Pathol. 2017a;10(7):7481-91.

Zhang Y, Li X, Hou Y, et al. The IncRNA XIST exhibits oncogenic properties via regulation of miR-449a and $\mathrm{BCl}-2$ in human non-small cell lung cancer. Acta Pharmacol Sin. 2017b;38(3):371-81.

Zhang Q, Chen B, Liu P, et al. XIST promotes gastric cancer (GC) progression through TGF- $\beta 1$ via targeting miR-185. J Cell Biochem. 2018;119(3):2787-96.

Zhang X, Pan S, Wang A, et al. Long non-coding RNA (IncRNA) X-inactive specific transcript (XIST) plays a critical role in predicting clinical prognosis and progression of colorectal cancer. Med Sci Monit. 2019a;25:6429-35.

Zhang J, LiW, Yang Y, et al. LncRNA XIST facilitates cell growth, migration and invasion via modulating $\mathrm{H} 3$ histone methylation of DKK1 in neuroblastoma. Cell Cycle. 2019b;18(16):1882-92.

Zhang R, Wang Z, Yu Q, et al. Atractylenolide II reverses the influence of IncRNA XIST/miR-30a-5p/ROR1 axis on chemo-resistance of colorectal cancer cells. J Cell Mol Med. 2019c;23(5):3151-65. 
Zhang M, Wang F, Xiang Z, et al. LncRNA XIST promotes chemoresistance of breast cancer cells to doxorubicin by sponging miR-200c-3p to upregulate ANLN. Clin Exp Pharmacol Physiol. 2020;47(8):1464-72.

Zhao C, Bai X, Hu X. Knockdown of IncRNA XIST inhibits hypoxia-induced glycolysis, migration and invasion through regulating miR-381-3p/ NEK5 axis in nasopharyngeal carcinoma. Eur Rev Med Pharmacol Sci. 2020:24(5):2505-17.

Zhao H, Wan J, Zhu Y. Carboplatin inhibits the progression of retinoblastoma through IncRNA XIST/miR-200a-3p/NRP1 axis. Drug Des Dev Therapy. 2020;14:3417-27.

Zheng H, Zhang M, Ke X, et al. LnCRNA XIST/miR-137 axis strengthens chemo-resistance and glycolysis of colorectal cancer cells by hindering transformation from PKM2 to PKM1. Cancer Biomark. 2020. https://doi. org/10.3233/CBM-201740.

Zheng W, Li J, Zhou X, et al. The IncRNA XIST promotes proliferation, migration and invasion of gastric cancer cells by targeting miR-337. Arab J Gastroenterol. 2020a;21(3):199-206.

Zhou X, Xu X, Gao C, et al. XIST promote the proliferation and migration of non-small cell lung cancer cells via sponging miR-16 and regulating CDK8 expression. Am J Transl Res. 2019;11(9):6196-206.

Zhou K, Yang J, Li X, et al. Long non-coding RNA XIST promotes cell proliferation and migration through targeting miR-133a in bladder cancer. Exp Ther Med. 2019;18(5):3475-83.

Zhu H, Zheng T, Yu J, et al. LncRNA XIST accelerates cervical cancer progression via upregulating Fus through competitively binding with miR-200a. Biomed Pharmacother. 2018a;105:789-97.

Zhu J, Zhang R, Yang D, et al. Knockdown of long non-coding RNA XIST inhibited doxorubicin resistance in colorectal cancer by upregulation of miR-124 and downregulation of SGK1. Cell Physiol Biochem. 2018b;51(1):113-28.

Zou L, Chen F, Xia R, et al. Long noncoding RNA XIST regulates the EGF receptor to promote TGF- $\beta 1$-induced epithelial-mesenchymal transition in pancreatic cancer. Biochem Cell Biol. 2020;98(2):267-76.

\section{Publisher's Note}

Springer Nature remains neutral with regard to jurisdictional claims in published maps and institutional affiliations.

Ready to submit your research? Choose BMC and benefit from:

- fast, convenient online submission

- thorough peer review by experienced researchers in your field

- rapid publication on acceptance

- support for research data, including large and complex data types

- gold Open Access which fosters wider collaboration and increased citations

- maximum visibility for your research: over 100M website views per year

At BMC, research is always in progress.

Learn more biomedcentral.com/submissions 